\title{
Somatic transposition in the Drosophila intestine occurs in active chromatin and is associated with tumor suppressor gene inactivation
}

Katarzyna Siudeja ${ }^{\star 1,2, ~ \#, ~ M a r i u s ~ v a n ~ d e n ~ B e e k ~}{ }^{* 1,2}$, Nick Riddiford ${ }^{1,2}$, Benjamin Boumard ${ }^{1,2}$, Annabelle Wurmser $^{1,2}$, Marine Stefanutti ${ }^{1,2}$, Sonia Lameiras ${ }^{3}$, Allison J. Bardin ${ }^{1,2, \#}$

1 Institut Curie, PSL Research University, CNRS UMR 3215, INSERM U934, Stem Cells and Tissue Homeostasis Group, Paris, France.

2 Sorbonne Universités, UPMC Univ Paris 6, Paris, France

${ }^{3}$ ICGex Next-Generation Sequencing platform, Institut Curie, PSL Research University, 75005 Paris, France

${ }^{*}$ Equal contribution

\# Authors for correspondence (katarzyna.siudeja@curie.fr, allison.bardin@curie.fr)

Twitter:@BardinLab

\begin{abstract}
Transposable elements (TEs) play a significant role in evolution by contributing to genetic variation through germline insertional activity. However, how TEs act in somatic cells and tissues is not well understood. Here, we address the prevalence of transposition in a somatic tissue, exploiting the Drosophila midgut as a model system. Using whole-genome sequencing of in vivo clonally expanded gut tissue, we map hundreds of high-confidence somatic TE integration sites genome-wide. We show that somatic retrotransposon insertions are associated with inactivation of the tumor suppressor Notch, likely contributing to neoplasia formation. Moreover, by applying Oxford Nanopore long-read sequencing technology, as well as by mapping germline TE activity, we provide evidence suggesting tissue-specific differences in retrotransposition. By comparing somatic TE insertional activity with transcriptomic and small RNA sequencing data, we demonstrate that transposon mobility cannot be simply predicted by whole tissue TE expression levels or by small RNA pathway activity. Finally, we reveal that somatic TE insertions in the adult fly intestine are found preferentially in genic regions and open, transcriptionally active chromatin. Together, our findings provide clear evidence of ongoing somatic transposition in Drosophila and delineate previously unknown underlying features of somatic TE mobility in vivo.
\end{abstract}




\section{Introduction}

Transposable elements (TEs) are DNA sequences that shape evolution through their capacity to amplify and mobilize, thereby altering the structural and regulatory landscape of the genome. Numerous mechanisms restrict the mobility of TEs and therefore, their mutagenic potential. In germline and somatic cells, TE silencing is achieved by chromatin modifications and small RNA-directed degradation of TE transcripts (Molaro and Malik 2016; Deniz et al. 2019; Cosby et al. 2019). The escape of TEs from silencing allows their propagation in the genome. While de novo TE insertions in the germline are relatively easy to detect as they result in heritable genomic changes that can be detected through sequencing, TE mobility in somatic cells is more difficult to study. Indeed, the heterogeneity of transposition events within somatic tissues imposes technical challenges as rare TE insertion events affecting a subpopulation of cells often fall below the limits of detection. Thus, the degree to which TEs evade silencing and contribute to somatic genome alteration is much less well understood in developing and adult tissues.

Nonetheless, evidence for active somatic transposition has been recently mounting. Reporters of transposon activity suggested TE mobility in neuronal lineages in human, mouse and Drosophila (Muotri et al. 2005; Coufal et al. 2009; Li et al. 2013; Macia et al. 2017; Chang et al. 2019). Additionally, recent use of an engineered gypsy retrotransposon trapping cassette in flies suggested that somatic transposition could also occur in non-neuronal tissues such as the fat body (Jones et al. 2016; Wood et al. 2016) or the intestine (Sousa-Victor et al. 2017). However, a major drawback of using such reporters is that these reporter cassettes could be inactivated by other means than a TE insertion. In addition, the available transgenic lines only report a limited number of TE families. Finally, results obtained with engineered reporters may not necessarily reflect the activity of endogenous elements encoded in the genome.

Genomic sequencing has provided some direct evidence for endogenous somatic retrotransposition though it has almost exclusively focused on the retrotransposition of LINE1 (L1) elements in human cancers (Lee et al. 2012; Solyom et al. 2012; Tubio et al. 2014; Rodić et al. 2015; Doucet-O'Hare et al. 2016; Tang et al. 2017; Rodriguez-Martin et al. 2020) or in human and rodent neuronal tissues (Baillie et al. 2011; Evrony et al. 2012; Upton et al. 2015). However, the first reports of high L1 transposition frequencies in mammalian brains were later shown to be overestimated due to artefacts of sequencing methodology and data analysis (Evrony et al. 2016). Similarly, in Drosophila, endogenous somatic TE mobility remains controversial as sequencing performed on populations of adult fly neurons failed to identify true insertions among multiple technical artefacts (Perrat et al. 2013; Treiber and Waddell 2017). Thus, the true extent to which diverse classes of TEs affects genomes of somatic tissues remains to be addressed. Moreover, due to low numbers of somatic insertions recovered thus far from non-cancerous conditions, integration site preferences of TEs in normal tissues in vivo are not well understood. Finally, a genetically amenable model system to reliably study somatic transposition is currently lacking. 
We have previously established the Drosophila midgut as a model system to address the prevalence of somatic mutation in an adult self-renewing tissue (Siudeja et al. 2015). The fly midgut is maintained by a population of intestinal stem cells (ISCs) that divide to self-renew and give rise to two differentiated cell types: absorptive enterocytes (ECs) and secretory enteroendocrine cells (EEs) (Micchelli and Perrimon 2006; Ohlstein and Spradling 2006). Our previous study demonstrated that ISCs acquire genetic mutations including deletions and complex rearrangements, which have important physiological impact on the tissue (Siudeja et al. 2015).

Here, we make use of the fly intestine to demonstrate the contribution of TEs to the somatic genetic variation of an adult tissue. Using whole-genome sequencing of clonally expanded gut neoplasia, we reveal ongoing somatic retrotransposition in the fly midgut. We identify de novo TE insertions in the tumor suppressor gene Notch, likely contributing to its inactivation and neoplasia formation. Additionally, we apply Oxford Nanopore long-read sequencing of non-clonal healthy adult tissues to provide evidence of tissue-specific differences in retrotransposition. Based on hundreds of high-confidence de novo transposition events identified genome-wide, we uncover nonrandom distribution of somatic TE insertion sites in the gut tissue. Transposition occurs throughout the genome and somatic insertions are enriched in genic regions as well as active, enhancer-like chromatin. Overall, by providing direct DNA sequencingbased evidence for de novo somatic insertions, we uncover novel features of their in vivo biology. 


\section{Results}

\section{Somatic TE insertions in the Notch gene identified in spontaneous intestinal neoplasia}

We have previously shown that somatic mutations occur frequently in intestinal stem cells (ISCs) and that the spontaneous inactivation of a tumor suppressor Notch in male adult ISCs drives the clonal expansion of mutant cells and formation of gut neoplasia (Siudeja et al. 2015). Neoplasia can be easily distinguished by the clonal accumulation of two intestinal cell types: ISCs expressing Delta and enteroendocrine cells (EEs) marked by Prospero. Our initial sequencing analysis of clonal neoplasia isolated from ProsGAL4 UAS-2xGFP (hereafter abbreviated as Pros $>2 X G F P$ ) male flies, revealed inactivation of Notch by large deletions or complex genomic rearrangements (Siudeja et al. 2015). In order to expand this analysis and better characterize distinct types of somatic mutations that impact adult ISCs, we generated a large dataset of whole-genome paired-end Illumina sequencing of an additional 30 clonal neoplasia from the same genetic background, as well as four clonal neoplasia from DeltaGAL4 UAS-nlsGFP male flies (hereafter abbreviated as Delta>nlsGFP), for a total of 37 clonal samples and matched control head DNA sequenced with an average of $47 x$ coverage (Fig $1 A$ and

Supplemental Table 1). These data are also analyzed by companion paper that addresses structural variation in the same model system (see Methods and also Riddiford, et al, submitted). As expected, a majority of clonal samples showed evidence for inactivation of the Notch pathway by somatic deletions or complex rearrangements (for details see Riddiford et al, submitted). Interestingly, four samples (P15, P47, P51 and D5) did not harbor any other mutation that could explain the clonal expansion, but showed evidence of somatic transposable element sequence inserted in Notch (Fig 1B and C). Thus, the TE insertions were most likely causative of the clonal expansion and Notch mutant phenotype. Strikingly, in sample P15, we observed two integrations within Notch (Fig 1C), with one of the two events having more sequencing reads supporting the insertion than the other, suggesting that the first insertion inactivated Notch, while the second one occurred later during the clonal expansion. All candidate insertions were supported both by clipped-reads mapping partially to a TE and partially to Notch, and paired-end reads where one mate-pair is TE anchored and the other is mapped to Notch (Fig 1B). Among the five candidate insertions identified, three were within the UTR regions of the gene and two TE integrations were in intronic sequences (Fig 1C). For all cases described, no read evidence was found for an insertion in the matched head DNA controls. Thus, TE insertions appeared specific for the clonal gut DNA, suggesting they occurred in somatic gut tissue (Fig 1B).

To validate the Notch TE insertions, we designed primer pairs flanking the identified insertion sites and performed a full-length PCR amplification using the original genomic DNA as a template (Fig 1D). Out of three candidate TE insertions tested, all passed PCR validation, showing $\sim 8 \mathrm{~kb}$ insertions likely representing full length TEs. All insertions were amplified only from the clonal neoplastic DNA and not the DNA of matched control tissues from the same fly, confirming that these were true neoplasia-specific somatic TE insertions. Finally, we could further confirm by Sanger sequencing the integration of a rover LTR retrotransposon in a reverse orientation in the 3'UTR of Notch in the sample P47 (Fig 1E). 
Altogether, these data revealed that TEs actively transpose in the adult midguts. Importantly, TE insertion can occur into the Notch tumor suppressor gene in stem cells, likely driving neoplastic growth in male flies.

\section{Retrotransposition occurs genome-wide in the fly midgut}

Having identified that TEs are mobile in the fly midgut and likely inactivate the Notch locus, we then aimed to address the prevalence of somatic transposition on a genome-wide scale. To precisely map somatic TE insertions from our short-read sequencing data, we developed a dedicated pipeline (Fig $2 A$, details in the methods section) and applied it to neoplastic and matched control samples. Briefly, we detected all split-read alignments and discordant alignments supporting TE insertions, isolated clusters of such alignments genome-wide and performed local assembly of the aligned sequence clusters to identify insertion sites with single base-pair resolution. We then filtered for sample-specific calls using all head samples as a panel of normal controls to ensure that somatic, and not germline, insertions are called while retaining the ability to discover recurrent insertions. Finally, we included a manual validation step to exclude any false positives (details in the Methods section).

For further analysis, we retained only insertions bearing a target site duplication (TSD) as a footprint of transposition-dependent events. TSDs are short, identical, duplicated sequences generated on both sides of a TE insertion as a consequence of a staggered endonuclease cut of the target DNA (Feng et al. 1996). We identified a total of 674 (median of 15 per clonal genome) somatic insertions with TSDs from the Pros>2XGFP background and 97 (median of 23 per clonal genome) integrations in the Delta>nlsGFP samples, all of which were private to gut clonally amplified samples and not present in the matched control DNA, or any of the controls (Fig 2B, Supplemental Table S1). Only one gut clonal sample (P13) showed no de novo insertions, however this sample was excluded due to low read complexity. In both genetic backgrounds, a great majority of identified insertions were retrotransposons (Fig 2C), suggesting that this TE class is the most mobile in the gut tissue. In the Pros>2XGFP background the most prominent were insertions of rover elements (487 insertions), followed by copia (102 insertions), diver (7 insertions), blood (5 insertions), roo (4 insertions), as well as sporadic insertions of other LTR TE families (Fig 2C). Among non-LTR retroelements, we identified de novo integrations of LINE-like retrotransposons, including 32 de novo insertions of I-elements, four F-element insertions and rare insertions of $X$, jockey, Doc, Rt1b, and G-elements. Insertions of terminal inverted repeat (TIR) DNA elements, such as 1360, hobo or S-element (1 integration each) were infrequent (Fig 2C). Finally, two somatic integrations of foldback elements, belonging to a distinct class of TEs described in Drosophila (Truett et al. 1981), were also identified (Fig 2C). In the Delta>n/sGFP background we mapped 16 insertions of copia elements, 10 roo integrations, followed by 297 (9 insertions), opus (8 insertions), mdg1 and Tabor (7 insertions each) as well as other LTR TE families (Fig 2C). Non-LTR LINE-like elements also generated de novo integrations of Doc, F, jockey, R1A1, Rt1b, baggins and Ielements. Rare integrations of DNA TIR class TEs, such as 1360, pogo, Tc1-2, Tc3 or S-element, were also found. Differences in mobile TE families can be noticed between the two genotypes. The most active LTR element family in the Pros>2XGFP background, rover, as well as LINE-like I-elements, were 
not detected to mobilize in Delta>nlsGFP clonal samples. In contrast, 297, opus, mdg1 and Tabor LTR elements that mobilized in the Delta>nIsGFP genetic background were rarely detected to mobilize in Pros $>2 X G F P$ samples. Nevertheless, some TE families, including the second most active element, copia LTR, mobilized in both genetic backgrounds. Thus, these data suggest that the repertoire of somatically mobile TEs depends on the genetic background.

To further confirm if the identified TE insertions were indeed true transposition events, we analyzed somatic TSDs for all TE families which produced at least six de novo insertions and compared these with known germline TSDs. Most LTR elements generated short TSDs with a median length below 10 base-pairs ( 5 bp for rover, copia, roo, 297 and diver, and 3 bp for blood), consistent with TSD lengths reported previously for germline insertions of LTR elements (Dunsmuir et al. 1980; Linheiro and Bergman 2012) (Fig 2D). Three LTR elements, opus, Tabor and $m d g 1$, produced unexpectedly long TSDs with a median of 23,26 and 25 bp respectively, in contrast to 4 bp reported previously for these elements (Linheiro and Bergman 2012). However, with relatively low numbers of somatic insertions of these TE families, it is difficult to conclude if this discrepancy with previously published reports could be biologically relevant. TSDs generated by LINE-like elements were, in general, less strictly defined but centered above 10bp (median of 12, 25 and 11 for I-, F- and Doc-elements respectively, Fig 2D), in agreement with previous reports (Bucheton et al. 1984; Sang et al. 1984; Driver et al. 1989; Berezikov et al. 2000). Finally, we searched for target site motifs for the most represented TEs. Highly significant (AT)-rich target site sequence motif was identified for the rover LTR-element reflecting non-random integration (Fig 2E). Although there are no previous reports about target site preferences of rover elements, TEs from closely-related classes (such as 297 or 17.6) show similar (AT)-rich target motives (Whalen and Grigliatti 1998; Bowen and McDonald 2001; Linheiro and Bergman 2012). The second most mobile element in our datasets, copia, did not show target site preference, which is consistent with previous reports from germline analyses (Dunsmuir et al. 1980). Altogether, our data shows that genome-wide somatic TE integration sites have similar characteristics to germline insertions. This lends further support to the detected TE insertions in the gut being true somatic transposition events, rather than random DNA integrations or products of chimeric reads.

Notably, using our detection criteria, we identified only rare somatic TE insertions in the head samples of both genotypes sequenced (median of 2 insertion/sample in Pros>2XGFP heads and 2.5 insertions/sample in DI>n/sGFP heads, Supplemental Fig S1A and B). However, the frequency of transposition between gut and head samples cannot be directly compared in this assay. Indeed, the head is a heterogeneous cell population, therefore somatic transposition in a few cells of the head would be below the detection level in our analyses. In contrast, the intestinal neoplasia are clonal expansions of single ISC genomes, increasing likelihood of detecting TE insertions. Accordingly, the rare somatic insertions identified in head samples had only a few clipped and mate-pair supporting reads, reflecting that these were likely rare events present in limited numbers of cells (Supplemental Fig S1C). This difficulty to detect TE insertions in non-clonal fly head DNA is also in agreement with recently published data (Treiber and Waddell 2017). Because single cell-insertions are unlikely to be detectable in our 
assay, we believe that the identified head insertions occurred likely during brain development leading to a small clone of cells harboring the TE insert, rather than in an adult fly brain, which is post-mitotic. Alternatively, they could represent rare but recurrent insertions arising independently in multiple cells of the adult fly brain.

Overall, we conclude that somatic retrotransposition in the fly midgut is not limited to the Notch locus, but occurs genome-wide. LTR-elements are the most active, while LINE-like retrotransposons mobilize less frequently. Although TE families identified as the most mobile can differ between fly strains, our data suggests that retrotransposons are frequently active in gut tissue.

\section{TE insertions arise before and after the clonal expansion}

To better understand when somatic transposition occurs in the fly gut, we then used allele frequency estimates to time genome-wide de novo integrations identified in clonal samples relative to the event inactivating Notch and initiating the clonal expansion (Fig $3 A$ ). The allele frequency is the ratio of sequencing reads supporting and opposing any given insertion. A TE insertion could arise before the onset of neoplasia, either during development or in the young adult gut, and be present in some cells of the normal tissue. Upon the Notch pathway inactivation, a stem cell would initiate clonal expansion and, at the time of analysis, the insertion would be present in all clonal cells as well as neighboring "normal" cells isolated for sequencing along with the clone. Thus, assuming the observed allele frequency represents the true population allele frequency, such a variant would present estimated allele frequency equal or greater than the Notch mutation. Alternatively, transposition could also occur after the clonal expansion, in which case such insertion would then be present in a fraction of cells of the clone and show estimated allele frequency lower than that of the Notch pathway inactivating mutation.

In both genetic backgrounds sequenced, we uncovered TE insertions causative for Notch inactivation and initiating neoplasia formation, indicating pre-clonal TE mobility (Fig 1C). In the Pros $>2 x G F P$ background $63.5 \%$ of genome-wide somatic integrations showed allele frequency higher than the Notch pathway inactivating event in the same sample (Fig $3 B$ ). These insertions were thus likely present in the tissue before the onset of neoplasia and occurred either during gut lineage specification in development or in young adult life. The remaining insertions (36.5\%) were of lower allele frequency than Notch pathway inactivating events, indicating that transposition continued to occur within clonal populations of cells after the initiation of a neoplasia (Fig 3B). High- and low-allele frequency insertions were detected for all TE families for which insertion counts were high enough to allow such analysis (Fig 3C). The LTR-elements rover and copia inserted equally often before and after the neoplasia initiating event. In contrast, LINE-like I-element integrations were moderately, but significantly, enriched among variants with allele frequencies higher than the Notch events, suggesting that this TE family might be more active during development in the precursor lineage of the gut or in the normal adult guts prior to the onset of neoplasia. In contrast to Pros $>2 X G F P$ neoplastic clones, insertions in Delta>nIsGFP samples were largely subclonal to the neoplasia initiating events $(96.8 \%)$, indicating that in this genetic background a majority of detected TE integrations occurred in the adult gut after the onset of neoplasia 
(Fig 3B). However, the remaining few insertions, including one in Notch, likely causative of Notch inactivation, indicate that pre-clonal mobility also occurred in this genetic background.

Together, these results imply that somatic retrotransposition in the fly gut can drive inactivation of a tumor suppressor Notch, and that it can occur before and after the clonal expansion. This suggests that retrotransposon activity is not restricted to neoplasia and can act through adult life in the gut and perhaps also during development.

\section{TE expression levels do not predict their mobility}

The expression and activity of LINE1 (L1) elements, the only somatically active TEs identified to date in the human genome, are increased in different tumor types (reviewed in (Burns 2017)). Our allele frequency analysis suggested that many TE insertions preceded clonal expansion, suggesting that TEs were active in a normal tissue. Nevertheless, we wished to address whether the initiation and clonal expansion of gut neoplasia could lead to transposable element deregulation. Thus, we asked if inactivation of Notch in a stem cell, which leads to a clonal neoplasia, could also cause increased TE expression. To do this, we compared TE expression levels in previously published RNA sequencing data of FACS-sorted wild-type and Notch RNAi knockdown ISCs (Patel et al. 2015). Expression of most TEs was not affected upon Notch knock-down (Supplemental Fig S2). Five TE families were significantly upregulated and four families were downregulated in Notch knock-down cells. However, changes were of low magnitude (fold change ranging from 1.5 to 3.5 ) and none of the differentially expressed TEs in Notch RNAi ISCs overlapped with the mobile TE classes identified in our assays (Supplemental Fig S2). Even though this data set comes from a different genetic background than the one we used to isolate neoplastic clones, the results suggest that neither inactivation of the Notch pathway nor hyperproliferation of the gut tissue are sufficient to strongly deregulate TE expression.

We next asked whether there was a correlation between expression levels of TE classes and their mobility. To do so, we performed RNA sequencing of normal (non-neoplastic) Pros $>2 x G F P$ midguts and compared this with our data on de novo TE insertions (Fig 4A). Most TEs showed very low (TPM, transcript per million $<1)$ or low $(1<$ TPM $<5)$ transcript levels. Eight TE families ( 7 LTR and 1 LINE-like element) showed TPM values above 5. The most highly expressed elements, copia-LTRs, contributed $73.5 \%$ of all TE-mapping transcripts (TPM=481), while the second and third most highly expressed TEs, springer and accord, represented $6.3 \%$ and $2 \%$ respectively. copia was also the second most mobile element identified in this genetic background. Strikingly, mobility did not correlate with TE expression levels, as the most active LTR-elements rover as well as the most active LINE-like Ielements, were both very lowly expressed in the tissue $($ TPM $<1)(\boldsymbol{F i g} \mathbf{4 A})$. For certain moderately expressed TE families, such as LTR element, invader1, or the LINE-like element, Juan, the canonical TE sequence was only partially covered, suggesting that full length, transposition-competent copies were not transcribed (Fig 4B). Notably, these data show that, at the tissue-wide scale, steady-state levels of TE transcripts are not good predictors of TE mobility. 
On the post-transcriptional level, somatic control of TEs is mostly achieved by the endogenous siRNA (endo-siRNA) pathway (Chung et al. 2008; Czech et al. 2008; Ghildiyal et al. 2008). Our gut transcriptome analysis showed that siRNA pathway genes, Argonaute 2 (AGO2), Dicer-2 (Dcr-2), loquacious (loqs) and $r 2 d 2$ were expressed, suggesting that this pathway is functional in the fly midgut (Fig 4C). This was further confirmed by sequencing of the gut small RNA fraction, which detected short 21-nucleotide sense and antisense reads complementary to TEs, as expected for the Drosophila siRNAs (Fig 4D). We found that siRNA levels were not directly proportional to the TE transcript levels (Fig 4E). The five active TE families (rover, copia, I-element, diver and F-element), responsible for 93.8 $\%$ of all insertions, showed low levels of 21 -nt antisense siRNAs. Thus, post-transcriptional silencing by siRNAs of these elements could be inefficient. Nevertheless, low siRNA levels were not a prerequisite for mobility, as we also detected de novo insertions of TEs (including blood and roo) that had abundant siRNAs present (Fig $4 D$ and $E$ ). Importantly, this implies that low levels of siRNAs could allow for the somatic mobility of some TEs, while other TEs retain their ability to mobilize even in the presence of abundant siRNAs.

Recent reports suggested that the piwi-interacting RNA (piRNA) pathway, known to control TEs in the gonads (Brennecke et al. 2008; Chambeyron et al. 2008), could also play a role in somatic TE silencing (Perrat et al. 2013; Jones et al. 2016; Sousa-Victor et al. 2017). However, it remains to be proven whether piRNAs are indeed produced in somatic tissues. We did not detect abundant 23-30 nucleotidelong RNAs (characteristic of piRNAs) in the analyzed gut small RNA samples (Fig 4D). Thus, if piRNAs are produced in the gut, they are at low levels and were under our detection limit. In contrast, 23-30 nucleotide-long RNAs with a typical "ping-pong" signature, indicative of piRNAs (Brennecke et al. 2007; Gunawardane et al. 2007), were easily detected in ovary controls of the Pros $>2 x G F P$ females. These were complementary to all TEs, including somatically active TE families (rover, copia, and I-element), suggesting that piRNA-mediated TE silencing of these TEs was properly established in the female germline (Supplemental Fig S3A and $\boldsymbol{B}$ ). There were also no significant differences in ovary piRNA levels between two parental stocks (ProsGAL4 and UAS-2xGFP) used to obtain the Pros $>2 x G F P$ flies (Supplemental Fig S3C and D). Thus, the observed somatic TE activity could not be explained by differences in active TEs between the parental genotypes, as previously documented in Drosophila dysgenic crosses (Brennecke et al. 2008).

Altogether, we find that in the fly gut neoplastic transformation is not necessary for TE expression and that many TE families are transcribed in the normal gut tissue. At the tissue-wide scale, TE RNA levels do not correlate with somatic mobility and even very low transcript levels can be sufficient for active transposition. Although post-transcriptional control by the siRNA pathway is in place, some retrotransposons escape this control and mobilize in the tissue.

\section{Tissue-specific transposition}

To further address TE mobility in normal tissues without a clonal expansion, we decided to apply longread sequencing to bulk genomic DNA obtained from either pooled midguts or pooled heads from the 
same individuals of the Pros $>2 x G F P$ background. High molecular weight genomic DNA was sequenced to $85 x$ coverage using the Oxford Nanopore Technology (ONT). We then detected all full-length, nonreference and tissue-specific TE copies entirely contained in a sequencing read (Fig $5 \boldsymbol{A}$ ). Considering that in the absence of clonal expansion, any somatic insertion would be very rare in sequencing of bulk DNA, we extracted only insertions supported by a single read ("singletons") and classified them as potentially somatic. To help to exclude germline variants, we eliminated all insertions detected in both gut and head DNA pools. Finally, as in the short-read sequencing datasets, we retained only insertions generating a valid TSD, a footprint of active transposition. Among all singleton insertions, rover LTRelements was detected the most frequently in gut DNA (152 insertions) (Fig 5C). Importantly, the fact that rover singleton insertions are the most frequent in both the long-read data as in the lllumina shortread data, supports the notion that these are likely somatic de novo insertions. Additionally, mapped rover insertions found in long-read ONT data had identical AT-rich target site motifs to those identified in clonal samples with Illumina sequencing (Fig $2 E$ and $5 C$ ). Similar to the short-read Illumina sequencing, singleton reads from the gut were also found containing other LTR-elements (such as springer, copia, roo), LINE-like elements (Doc-, I-element) and DNA TIR elements (hobo, Bari1, Selement) (Fig 5C). While a total of 191 singleton insertions, representing putative de novo integration events, were recovered from the gut DNA, 24 singletons were also found in the head DNA. This suggests that somatic TE mobility occurs both in the gut as well as in the head, further supporting our findings of rare inserts in the head from the short-read sequencing data (see Supplemental Fig S1). Interestingly, with the exception of singleton insertions from roo LTR-elements, almost all singletons from other TE families were specifically found in one of the two tissues, suggesting that TE family activity may be tissue-specific. Singletons from LTR-elements rover, springer and copia, contributing together $82.8 \%$ of all putative somatic insertions, were found only in the gut tissue. Singletons of other elements (such as Stalker2), although rarer, appeared specific to the head DNA (Fig 5C). This suggest that the repertoire of somatically active TEs may differ between different tissues.

To gain further insight into the tissue-specificity of transposition, we then asked if the same TEs were mobile in the germline, leading to heritable de novo insertions. To do so, we sequenced 18 individual flies from the progeny of Pros>2xGFP parents (Supplement Figure S4A) and detected de novo germline insertions present in any of the progeny and not in the parents. We discovered 3 de novo foldback element insertions. Of note, we found no germline de novo insertions of any of the TE families found active in the somatic cells of the gut. This was in agreement with the analysis of small RNA fractions from ovaries indicating that somatically mobile TEs were properly silenced in the germline (Supplement Fig S3A and B).

Altogether, with the use of long-read sequencing, we provide further evidence supporting active transposition in the normal fly intestine. Furthermore, our data comparing gut and head DNA as well as inherited de novo insertions, indicates that 1) distinct TEs can be active in the different somatic tissues and 2) active TEs may differ between the soma and the germline. 


\section{Somatic transposition is enriched in genes and regions of active enhancer-like chromatin}

The identification of hundreds of somatic TE insertions with base-pair resolution, allowed us to address whether somatic transposition acted uniformly throughout the genome or specifically affected particular genomic regions. To shed light on preferential landing sites of somatically active TEs, we analyzed genomic and chromatin features of the de novo somatic TE insertion sites.

Analyzing the genome-wide distribution of somatic TE insertions from the clonal gut samples in mappable regions of the genome revealed that integrations occurred broadly across Drosophila chromosomes (Fig $6 \boldsymbol{A}$ ). Importantly, somatic transposition was very frequent in genic regions. $78.5 \%$ of all candidate insertions fell inside annotated genes and an additional $5.6 \%$ targeted potential promoter sequences less than $1 \mathrm{~kb}$ upstream of genes. Although they were depleted from coding sequences, somatic TE integration sites were significantly enriched in introns and 3'UTRs (Fig 6B). Similar genic preference was evident in the singleton insertions identified by the long-read sequencing of normal gut DNA pools (Supplemental Fig S5), suggesting that it was not influenced by the clonal expansion. 82.3\% of all singleton insertions fell inside annotated genes and additional $6.2 \%$ in gene promoters. Importantly, in both data sets, we found insertions within or nearby (<500bp upstream of the TSS) genes with established roles in the regulation of gut and ISC homeostasis (Fig 6C, Supplemental Table S2 and S3). Apart from the insertions in Notch, which were selected for in clonal samples, we detected insertions in genes involved in the EGFR (pointed, EGFR), JNK (puckered), JAK/STAT (Stat92E), Wnt (frizzled), insulin (Insulin-like receptor) and VEGF (Pvf1-3) pathways, as well as chromatin modifiers (kismet, osa) and other regulators of ISC homeostasis. Some of the affected genes were hit multiple times (Fig 6C, Supplemental Table S2 and S3). It is possible that these genes are hot-spots for TE insertions, perhaps due to genome sequence or structure. Alternatively, these insertions could drive positive selection of the resulting cell lineage through promoting stem cell proliferation, resulting in their post-insertion enrichment. Thus, we find that somatic TE insertions are enriched in genic regions and frequently target genes, including those with important functions in the gut.

To further probe into integration preferences of somatic TE insertions, we investigated the overlap between candidate integration sites mapped in clonal (Fig 6D) and pooled gut samples (Supplement Fig S5B) with publicly available Drosophila modENCODE datasets profiling chromatin features and transcription factor binding sites (The modENCODE Consortium et al. 2010). Comparing clonal gut TE insertion sites of LTR-elements (rover and copia) with tracks from adult fly tissues revealed significant depletions from genomic regions enriched in silent chromatin features, such as methylated histone $\mathrm{H} 3$ (H3K9me2/3, H3K27me3), Heterochromatin Protein 1a (HP1a) or linker histone H1 (Riddle et al. 2011). Although less strongly, LTR-element insertion sites were also depleted from regions marked by H3K36me2/3, typically associated with exons of transcribed genes (Kharchenko et al. 2011). This negative correlation is agreement with the depletion of TE insertion sites from coding regions as documented above (Fig 6B). In contrast, we observed a strong positive correlation between LTRelement integration sites and genomic regions rich in acetylated histone H3 (H3K18ac, H3K27ac) and $\mathrm{H} 4$ (H4K8ac) as well as $\mathrm{H} 3 \mathrm{~K} 36 \mathrm{me} 1$, marks associated with active promoters, transcribed regions and 
enhancers (Kharchenko et al. 2011; Nègre et al. 2011). De novo insertions were also significantly enriched in genomic regions bound by LSD1/Su(var)3-3, a histone lysine-demethylase responsible for removing histone H3K4-methyl marks from active promoters (Shi et al. 2004; Stefano et al. 2007). The correlations for LTR-element insertions identified in clonal and pooled DNA samples were very similar (Fig 6DB and Supplement Fig S5B), suggesting that the distribution of somatic insertion sites was the same for normal and neoplastic tissue. Correlations of the LINE-like l-element insertion sites were much weaker than those obtained for LTR elements, and should be interpreted with caution due to a low total number of insertions (Fig 6D). However, the enrichment in H3K36me1 was also significant for this nonLTR TE family. Consistent with the insertion timing analysis implying that transposition could act preclonally and during development (Fig 3B), comparable enrichments and depletions were also found for short-read clonal (Supplement Figure S6) and long-read bulk (Supplement Figure S5B) gut sequencing data sets with the modENCODE tracks derived from Drosophila larval stages.

To confirm whether similar TE insertion site enrichment could be observed with gut-specific chromatin features, we used our recently published DamID profiles of chromatin factors in intestinal stem cells (Gervais et al. 2019) (Fig 6E). In agreement with the results obtained with the modENCODE datasets, somatic TE insertions from clonal gut samples were highly enriched in genomic regions bound by chromatin modifiers Kismet and H3K4 monomethyl-transferase Trithorax-related (Trr) as well as RNA polymerase II (Pol II) (rover, copia and I-element). All these factors were previously shown to map open, transcriptionally active chromatin (Marshall and Brand 2017; Gervais et al. 2019). Concurrently, somatic transposition sites of LTR-elements were strongly depleted from repressed chromatin domains bound by HP1 (a reader of histone H3K9me3) (Fig 6E). Finally, enrichment in chromatin domains bound by Kismet and Pol II, and depletion in HP1-bound sites were also significant for putative somatic singleton insertions identified in non-clonal gut DNA pools (Supplement Fig S5C), suggesting that the insertion site enrichment in active chromatin also occurred in normal tissues without clonal expansion.

Taken together, our data revealed non-random distribution of retrotransposon insertions sites in a somatic tissue in vivo. Somatic transposition more frequently affects genic regions of the genome and is enriched in open, transcriptionally active chromatin. 


\section{Discussion}

Our study provides a genome-wide view of how transposable elements mobilize in a somatic tissue. We show that endogenous retrotransposons mobilize in the fly gut and create de novo insertions genomewide. Somatic insertions preferentially affect genes and open chromatin, and they can occur in the Notch tumor suppressor, likely leading to clonal neoplasia.

\section{Somatic retrotransposition in the fly intestine}

By whole-genome sequencing of clonally expanded gut neoplasia, we were able to detect hundreds of high confidence retrotransposition events. Clonal expansion brings an advantage of amplifying in vivo any genetic variant otherwise present in a tissue with a very low frequency. A similar approach has previously been taken to demonstrate somatic transposition in human tumor samples. Indeed, retrotransposition was observed in many tumor types, including lung, ovarian, breast, colorectal, prostate, liver, pancreatic, gastric, esophageal and hepatic cancers (Iskow et al. 2010; Lee et al. 2012; Solyom et al. 2012; Shukla et al. 2013; Helman et al. 2014; Tubio et al. 2014; Doucet-O'Hare et al. 2015; Ewing et al. 2015; Paterson et al. 2015; Rodić et al. 2015; Scott et al. 2016; Schauer et al. 2018). Most of these studies failed to detect somatic integrations in matched healthy tissues, leading to the conclusion that transposition was likely limited to the cancerous state. However, careful analysis of gastro-intestinal and esophagus tissues did suggest that active retrotransposition occurring in normal cells, can undergo clonal expansion in a tumor context (Ewing et al. 2015; Doucet-O'Hare et al. 2016). Likewise, here we provide evidence that TEs are expressed and mobilize in a normal fly gut, without a prerequisite of neoplastic transformation. Indeed, our analysis of variant allele frequencies in clonal datasets suggests that transposition can occur in the adult tissue both in normal cells prior to the clonal expansion and in neoplastic cells undergoing uncontrolled proliferation. Studies of the mammalian brain have provided different pieces of evidence suggesting that somatic transposition can occur in the embryo, during neurogenesis as well as in mature neuronal cells (Evrony et al. 2012, 2015; Upton et al. 2015; Faulkner and Garcia-Perez 2017). Likewise, we detect TE insertions with variant frequencies higher than the event driving clonal expansion but with no supporting evidence in the head DNA, suggesting that somatic TE insertions could also be acquired during development and gut lineage specification as well as during adult life.

\section{Applying long-read sequencing to detect somatic transposition}

We provide further strong evidence for somatic transposition acting in a normal tissue with the use of long-read sequencing technology to assess rare, single read support insertions representing likely de novo TE integrations in bulk tissue DNA in the absence of clonal expansion. Indeed, this technology offers important benefits over classical short-read sequencing in mapping non-referenced TE insertions. It enables full-length detection of inserts within one sequencing read, resolving not only both ends but also the entire length of an insert. Moreover, it outperforms short-read technology in the analysis of lowcomplexity genomic regions where short-read mapping poses particular difficulty. Thus, rare somatic TE insertions can be detected from pooled DNA libraries, provided that robust controls are implemented 
to help to exclude germline variants in a population. Alternatively, the use of single individuals, rather than pooled tissues, would rule out germline variants. Thus, by the use of long-read sequencing we put forward a novel methodology for detecting somatic TE activity. A similar approach has very recently been proposed to perform epigenomic profiling and non-referenced TE mapping in human datasets (Ewing et al, bioRXiv 10.1101/2020.05.24.113068), further showing that long-read sequencing will certainly gain on popularity in the field. As our results obtained with this technology are highly consistent with our results obtained with short-read sequencing of neoplastic clones, we believe the singleton reads detected with long-read sequencing are very likely de novo somatic events. Thus, we reveal ongoing somatic transposition in the gut tissue using two complementary genomic approaches.

\section{Transposition across different tissues}

Historically, focus has been on somatic transposition in neuronal tissues, where TE mobility was proposed to contribute to functional differences between individuals (Erwin et al. 2014). Here, with the use of long-read sequencing, we detected putative somatic TE insertions in the gut and the head tissues. Our data suggest that TE families active in the gut are largely not mobile in the head of the same individuals, implying that the repertoire of mobile TEs might vary between different somatic tissues. Indeed, many studies have begun to uncover tissue- and cell-specific patterns of TE expression in human and model organisms (Mietz et al. 1992; Faulkner et al. 2009; Philippe et al. 2016; Deininger et al. 2017; Pehrsson et al. 2019; Chung et al. 2019; Ansaloni et al. 2019; Sanchez-Luque et al. 2019) (Treiber and Waddel, bioRxiv 10.1101/838045). However, in most cases, the lack of data on somatic insertions hinders the direct comparison between transcriptional activity and mobility. Importantly, here we show that in the gut tissue, TE transcript levels do not parallel insertional activity. However, we cannot exclude that active TEs could be expressed in a cell-type specific manner and that cell-type specific TE expression patterns could correlate better with the mobility. Nevertheless, our data suggest that caution should be taken when using transcript levels as a proxy for TE insertional activity. How additional factors, aside from those regulating RNA transcript levels, may contribute to tissue-specific somatic TE mobilization, remains to be determined.

Apart from gut vs head specificity, we also show that somatically active TEs were not detected to be mobile in germ cells. Thus, our data speaks against an overall deregulation and high retrotransposon activity, as previously documented in the germline of Drosophila dysgenic crosses (Kidwell et al. 1977; Pélisson 1981; Rubin et al. 1982) or in other rare genetic backgrounds with bursts of TE activity in the germline and soma (Gerasimova et al. 1985; Georgiev et al. 1990).

\section{TE insertions distribute non-randomly in the somatic genomes}

Mapping somatic retrotransposition insertions with base-pair resolution reveals enrichments in insertion site distribution of endogenous retrotransposons in vivo. Indeed, we show that the distribution of TE insertions is not random, but influenced by the genome sequence composition as well as by the local chromatin state. Gut retrotransposon insertions are enriched in transcriptionally active, enhancer-like chromatin, a bias that is similar to the insertion site preferences previously observed for the murine 
leukemia virus (MLV) and the PiggyBac transposon in human T-cell cultures (Gogol-Döring et al. 2016; Sultana et al. 2017, 2019). Similarly, recent analysis of TE insertion site preferences in human cancer genomes revealed enrichment in DNase hypersensitive open chromatin and depletion in histone H3K9me3-rich heterochromatin (Rodriguez-Martin et al. 2020). Our data uncover similar insertion site enrichments in vivo, not only in the context of neoplastic clones but also in a normal tissue. In the fly gut, as transposition acts in the context of renewing and dividing tissue, the uncovered insertion site distribution is likely a result of pre-insertion target site choice as well as post-insertion selection in the tissue, as previously demonstrated for de novo L1 insertions in human culture cells (Sultana et al. 2019). Negative selection probably contributed to the significant depletion of TE insertions in coding regions, as such insertions, presumably deleterious, would lead to their elimination from the tissue by clonal competition. Accordingly, insertion site enrichment in genic regulatory regions (UTRs and introns) could suggest a beneficial impact of transposition on the positive selection of cells with somatic TE insertions. Enrichment of de novo insertions in open chromatin could, at least partially, be explained by physical DNA accessibility. However, we cannot exclude that other, yet unknown mechanisms also act at the pre-insertion level to direct retrotransposition away from exons and silent chromatin but towards noncoding genic regions and active chromatin.

\section{Consequences of somatic transposition}

The impact of transposition on the biology of somatic tissues is under debate, as is its contribution to disease and aging (Faulkner and Garcia-Perez 2017; Chuong et al. 2017; Dubnau 2018). Here, we report evidence for somatic transposition with a functional impact on an adult tissue, by retrotransposon insertions into a tumor-suppressor gene Notch. As spontaneous neoplasia are isolated based on the characteristic Notch loss of function phenotype, and in those samples we found no other somatic events genome-wide that could explain inactivation of the Notch pathway (see also Riddiford et al, submitted), it is very likely that the somatic LTR retrotransposon insertions in Notch where indeed causative for neoplasia formation. Similarly, in mice somatic LTR element insertions causing oncogene or cytokine gene activation have been previously reported (Mietz et al. 1992; Howard et al. 2008). In human somatic de novo L1 retrotransposition activating oncogenic pathways has been documented in colorectal cancer (Miki et al. 1992; Scott et al. 2016) and hepatocellular carcinoma (Shukla et al. 2013), leading to a hypothesis that individual variation in somatically active elements could represent a novel form of cancer risk (Scott et al. 2016).

In addition to Notch inactivating events, many genome-wide retrotransposon insertions identified by us occurred in genic regions. We uncover exonic integrations, which would most likely lead to gene inactivation, as well as insertions in intronic or UTR sequences. Numerous examples of germline transposition show that TE insertions in non-coding genic regions can affect, both positively or negatively, target gene expression (Shen et al. 2011; Gong and Maquat 2011; Mateo et al. 2014; OngAbdullah et al. 2015; Ding et al. 2016; Van't Hof et al. 2016). Among the genes hit by retrotransposition in our datasets, some are directly implicated in the tissue physiology, including regulators of ISC proliferation and differentiation. As for the Notch gene, in male flies, an X-chromosome insertion would 
result in a modification of a single available gene copy. In contrast, when two alleles are present (autosomes and female $\mathrm{X}$ chromosome), a somatic insertion would likely inactivate only one of them, limiting the functional impact. However, such insertions could still result in hypomorphic phenotypes. Moreover, unaffected alleles could be lost due to secondary genetic events of loss of heterozygosity, which we have shown to occur frequently in the fly gut (Siudeja et al. 2015; Siudeja and Bardin 2017). Finally, apart from directly affecting coding regions, non-genic transposable element insertions occurring in open chromatin could contribute cis-regulatory elements acting on neighboring or even distant genes leading to gain-of-function or mis-expression, as previously demonstrated for germline transposition (reviewed in (Chuong et al. 2017)). Hence, we hypothesize that transposition acting on genes or regulatory regions in the ISCs could influence stem cell fitness. By doing so, TE mobility could influence the clonal selection in the tissue by eliminating some and favoring other stem cell genomes.

Since somatic transposition may have functional consequences and contribute to diseases or aging, understanding how somatic transposition is controlled and how tissue-specificity arises, is of keen interest. We provide evidence that Drosophila will be an insightful model system for addressing mechanisms of somatic TE control and physiological consequences of somatic transposition. 


\section{Materials and Methods}

\section{Experimental techniques:}

Drosophila stocks and husbandry

Pros $>2 X G F P$ adults were obtained by crossing $w$; $\operatorname{Pros}^{v 1}{ }^{1} G A L 4 / T M 6 B T b S b$ females (J. de Navascués) with w; UAS-2XGFP; males (Bloomington). DI>nlsGFP were obtained by crossing w; DIGal4/TM6TbHu (Wang et al. 2014) females with $w$; UAS-nlsGFP males (Bloomington). Flies were maintained on a standard medium at $25^{\circ} \mathrm{C}$ with a day/night light cycle. For crosses, 10-15 females were mixed with males in standard vials. Progeny were collected over 2-4 days after eclosion. Adults were aged in plastic cages (mixed males and females) (10 cm diameter, $942 \mathrm{ml}, 700-900$ flies/cage) with freshly yeasted food provided in petri dishes every $2-3$ days. Every 7 days, flies were transferred to clean cages.

Tissue isolation and short-read DNA sequencing

6-7-weeks-old Pros $>2 X$ XFP or DI>nlsGFP males were used to isolate spontaneous neoplasia from unfixed tissues. Clones where identified by accumulation of GFP-positive cells. The midgut region containing an estimated $30 \%-80 \%$ neoplastic cells was manually dissected and transferred immediately to a drop of the ATL Buffer (Qiagen) for subsequent DNA isolation. Neighboring control gut tissue as well as the fly head and the fly thorax were also dissected. Genomic DNA was isolated with the QIAamp DNA MicroKit (Qiagen) according to the manufacturer's protocol for processing laser-microdissected tissues. DNA quantity was measured with Qubit dsDNA High Sensitivity Assay Kit (Thermo Fisher Scientific). Genomic DNA libraries were prepared with the Nextera XT protocol (Illumina) using $0.6 \mathrm{ng}$ of starting material. Whole-genome $2 \times 100 \mathrm{bp}$ or $2 \times 150 \mathrm{bp}$ paired-end sequencing was performed on HiSeq_2500 or Novaseq 6000 (Illumina). Supplemental Table S1 provides basic sequencing statistics for all samples used in this study.

\section{$P C R$ validation of somatic TE insertions in Notch and Sanger sequencing}

We designed PCR primers down- and upstream of identified candidate insertions to amplify either a fulllength TE insertion or a short wild-type genomic DNA fragment. PCR was performed on $0.5-1 \mathrm{ng}$ of genomic DNA with the LongAmp Taq DNA Polymerase (New England Biolabs) using standard PCR reaction mix and long extension times $\left(9\right.$ minutes at $\left.65^{\circ} \mathrm{C}\right)$.

The following primers were used to generate Fig 1D:

R43-rover: GCAGCATTTGGTCCAAACGTT and ATAAAATGCGCCACAAGACGAG

R9-rover-ex: CGCGCAAGGATAATTGGATGG and GCGTAGTCTTATGGCCTAGTG

R9-rover-int: CCAGGCTGCAATTACTTTAATT and TGTAAAATGCAAGCGGAATGC

For Fig 1E, the DNA fragment containing the TE insertion was gel-purified and used as a template for a second PCR amplification using the same conditions as above. The amplified product was purified and Sanger sequencing was performed by Eurofins Genomics, using the following primers: GCAGCATTTGGTCCAAACGTT and CGTAGAGATTAGAGAATTACA.

\section{RNA and small RNA isolation and sequencing}

For RNA isolation, gut and head tissues from 1-week-old flies were dissected in cold, RNase-free PBS, transferred to $100 \mathrm{~L}$ of TRIzol Reagent (Thermo Fisher Scientific), homogenized with a plastic pestle and snap frozen in liquid nitrogen for storage at $-80^{\circ} \mathrm{C}$. Upon thawing, samples were further processed according to the TRIzol Reagent manufacturer's protocol. Purified RNA was treated with DNase (Ambion) for $1 \mathrm{hr}$ at $37^{\circ} \mathrm{C}$, further purified with phenol-chloroform extraction and isopropanol precipitation, and resuspended in RNase-free water. All samples had A260/280 ratios above 1.9 and A260/230 ratios above 2.0. RNA integrity was checked on Bioanalyzer (Agilent) using the Agilent RNA 6000 Nano Kit and concentrations were assayed with the Qubit RNA Broad Range Assay Kit (Thermo Fisher Scientific). For the transcriptome analysis, 700ng of total RNA was used to prepare libraries according to the TruSeq Stranded mRNA protocol (Illumina). Samples were processed in biological triplicates. 2X100 bp paired-end sequencing was performed on Novaseq (Illumina). Small RNA 
fractionation and sequencing was performed by Fasteris, SA (Geneva, CHE). Briefly, after 2S rRNA depletion and PAGE gel-sizing for 18-30-nt fragment size, libraries were prepared according to the TruSeq small RNA Kit (Illumina) and sequenced on NextSeq 500, in 1x50bp single-end mode. Samples were processed in biological duplicates.

High molecular weight genomic DNA isolation and long-read sequencing

For sequencing of non-neoplastic tissues, we isolated guts from 25-days-old female flies without any visible neoplasia along with the fly heads. Tissues were dissected in ice-cold, nuclease free PBS and snap-frozen in liquid nitrogen before DNA isolation. High molecular weight genomic DNA was isolated from pools of 60 guts or 60 heads with the MagAttract HMW DNA Kit (Qiagen) according to manufacturer instructions. gDNA was eluted with nuclease-free water. DNA integrity was verified on a $0.6 \%$ agarose gel and concentrations were measured with Qubit dsDNA Broad Range Assay Kit. All samples had A260/280 ratios above 1.8 and A260/230 ratios above 2.0. Libraries were prepared with 800ng of DNA following the 1D Genomic DNA by Ligation Protocol (SQK-LSK109, Oxford Nanopore Technologies). Sequencing was performed on MinION using R9.4.1 flow cells (Oxford Nanopore Technologies) and $48 \mathrm{hr}-$ long sequencing runs. Supplemental Table $\mathrm{S} 1$ provides basic sequencing statistics for all samples used in this study.

\section{Computational analysis:}

Putative TE insertion detection from short-read DNA sequencing

The following applies to all Illumina short read paired end DNA sequencing datasets. Adapter sequences were trimmed using fastp version 0.19 .5 (Chen et al. 2018). Trimmed reads were aligned to release 6.13 of the Drosophila Melanogaster reference genome (FlyBase) using bwa-mem version 0.7.17. bwamem parameters were default parameters, except for $-Y$ (use soft clipping for supplementary alignments) and -q (don't modify mapQ of supplementary alignments). Trimmed reads were also aligned to Drosophila melanogaster TE family consensus sequences (https://github.com/bergmanlab/transposons) using bowtie2 (Langmead et al. 2009) version 2.3.4.3. Duplicate reads were marked using picard markdup 2.18 .2 (http://broadinstitute.github.io/picard/). Genome alignments and TE alignments are inputs to the readtagger command of the readtagger package (https://github.com/bardin-lab/readtagger). readtagger writes SAM tags for alignments where either the alignment or the mate of the alignment also aligns to a TE or other non-reference genome sequence. Tags contain information about the alignment (TE reference, alignment start, alignment end, query start, query end, and alignment orientation), can be visualized in IGV and are used to locate potential non-reference TE insertions. Next, the findcluster command takes the tagged alignment files and iteratively splits and groups tagged reads within a distance that corresponds to the $95 \%$ interval of the insert distance into clusters based on their alignment orientation and clipped sequences, and annotates if any cluster shows signs of a target site duplication (TSD). These unfiltered clusters are further linked to soft-clipped sequences at the $5^{\prime}$ and $3^{\prime}$ ends of putative insertions, so that the presence of a particular clipped sequence at a given genomic position can be used as a proxy for determining whether reads are congruent with an insertion or not.

The output of the findcluster step is a GFF file containing putative insertions and soft-clipped sequences and their genomic position as well as a BAM file containing only aligned reads assigned to a cluster (this includes alignments that support an insertion and alignments that support the reference).

\section{Filtering putative TE insertions to obtain non-reference somatic TE insertions}

findcluster outputs for each sample were filtered to retain only insertions that contain both mate and split read support. These putative insertions were then processed with the confirm_insertions command of the readtagger package. This command takes as input a file containing pre-filtered putative insertions in GFF format, a set of all putative insertions from all samples and a set of insertions from all samples that can be considered a panel of normal. To detect somatic TE insertions for a particular tumor dataset the panel of normals are all head datasets. Inversely, to detect somatic head insertions, the panel of normal are all tumor datasets. confirm_insertions links insertions from all samples using overlapping 
clipped sequences, genomic location and the family of a putative TE insertion. Putative somatic TEs were those insertions that were not found within the panel of normal. We further required a valid target site duplication to be present. For each candidate somatic TE insertion we generated an IGV screenshot that includes 500 nucleotides up- and downstream of each insertion. Upon visual inspection of screenshots putative insertions that were likely incorrectly called due to imprecise annotation of either the putative insertion or the control insertion were discarded. The lists of all identified putative somatic insertions can be found in Supplemental Tables S4 (gut clonal samples) and S5 (head samples).

\section{Filtering putative TE insertions to obtain non-reference germline insertions}

To estimate the rate of germline transposition, datasets were analyzed as for somatic TE insertions, but we treated each family individually, where the panel of normal constituted all other families. The retained insertions were then private to the family being analysed.

\section{Comparison of somatic TE allele frequencies to neoplasia-initiating events}

Allele frequencies of somatic insertions were estimated from the total pool of read pairs that overlap a putative insertion. Allele frequencies of neoplasia-initiating events were taken from a companion paper addressing structural variation in the same model system (Riddiford, et al, submited). The exception to this were samples P15, P47 and D5, where somatic TE insertions in Notch were assigned as neoplasia initiating events. For sample P15, with two somatic insertion in Notch, the insertion with a higher allele frequency was set as the putative Notch inactivating event. We plotted the estimated allele frequency of insertions as dots and the tumor initiating event as a bar. We adjusted the allele frequency of TE insertions on autosomes by multiplying with a factor of 2 .

\section{Long-read sequencing data analysis}

Nanopore reads were basecalled using guppy version 3.2.4. Since read length and sequencing depth is not uniform for long-read datasets and this can affect the number of full-length TE insertions that are detectable, reads were normalized using the normalize_readsizes of the readtagger package. All analysed nanopore libraries therefore have the same read-size distribution and sequencing depth.

Reads were aligned to release 6.13 of the Drosophila melanogaster reference genome using minimap2 version 2.17 (Li 2018) with the $-\mathrm{Hk} 19$ preset for Nanopore reads and the $-\mathrm{Y}$ flag. Alignments with a mapping quality below 40 were discarded with samtools view. extract_variants from the readtagger package was used to check all soft-clipped or insert sequences for homology to TEs using mappy. Aligned positions around soft-clipped or insert sequences were written to a new alignment file along with a tag that describes the TE alignment. For soft-clipped sequences, a single aligned $\mathrm{N}$-nucleotide was written out together with the soft-clipped sequence. For inserts, the insert sequence was written out using 1 flanking $\mathrm{N}$ nucleotide at each site. Alignment files were then parsed into a tabular format and analysed to find unique full-length transposable element insertions using ipython notebooks available at https://github.com/bardin-lab/somatic-transposition-fly-intestine. The lists of all identified singleton insertions can be found in Supplemental Tables S6 (pooled gut samples) and S7 (pooled head samples).

\section{Motif analysis at integration sites}

Motifs were determined by extracting 10 flanking nucleotides upstream and downstream of each insertion using bedtools slopbed and bedtools getfastabed (Quinlan and Hall 2010) and running meme version 5.0.5 (Bailey et al. 2009) on the resulting multi-fasta file. meme parameters were -dna for DNA alphabet, -revcomp which checks the reverse complement for motifs, -pal for checking for palindromes and a motif width between 9 and 50 (-minw 8, maxw 50).

\section{Genome features enrichment analysis}

Pre-analyzed modencode datasets (The modENCODE Consortium et al. 2010) were downloaded and lifted over to release 6.13 of the Drosophila genome. DamID peaks (Gervais et al. 2019) were downloaded from GSE128941. Overlap was analyzed in ipython notebooks available at https://github.com/bardin-lab/somatic-transposition-fly-intestine. pybedtools fisher (Dale et al. 2011) was used to determine enrichment and significance of overlap. Correlations with $p$-value $<0.0001$ and - 
1.5>enrichment>1.5 were considered significant. The overlap between genes and TE insertion sites (Fig $6 \mathrm{C})$ was calculated with bedtools windowbed using $+/-500$ bp window size.

\section{RNAseq data analysis}

For RNAseq analysis reads were trimmed off their adapters using Atropos (Didion et al. 2017) and quasimapped against the Drosophila reference transcriptome (release 6.13, Flybase) supplemented with family-level TE sequences (https://github.com/bergmanlab/transposons) using Salmon version 0.14 .1 (Patro et al. 2017) and RPM values were reported in Fig 4. Differential expression analysis (Fig S2) was performed on previously published datasets (Patel et al. 2015) using DESeq2 (Love et al. 2014). TE transcripts with an adjusted $p$-value $<0.01$ were considered differentially expressed. We used the plot_coverage command of the readtagger package to create coverage plots.

\section{Small RNA analysis}

Sequencing adapters were trimmed using fastp version 0.19 .5 . Reads were aligned to family level TE sequences using HISAT2 (Kim et al. 2019) version 2.1.0. Size distributions were plotted using ipython notebooks. Ping-pong signatures were calculated using the Small RNA Signatures tool, version 3.1.0 (Antoniewski 2014).

\section{Data availability}

Datasets used for this study are available under the following accession numbers:

ArrayExpress, E-MTAB-3917 - whole-genome neoplasia/head control sequencing (samples P1-P6; (Siudeja et al. 2015))

NCBI, Bioproject, PRJNA641572 - whole genome neoplasia/head control sequencing (samples P7P66 and D1-D8; this study)

$X X X X X X X X$ - Nanopore ONT sequencing of pooled guts/heads, RNAseq of pooled guts and small RNAseq of pooled guts and ovaries (this study)

\section{Acknowledgements}

High-throughput sequencing has been performed by the ICGex NGS platform of the Institut Curie supported by the grants ANR-10-EQPX-03 (Equipex) and ANR-10-INBS-09-08 (France Génomique Consortium) from the Agence Nationale de la Recherche ("Investissements d'Avenir" program), by the Canceropole Ile-de-France and by the SiRIC-Curie program - SiRIC Grant «INCa-DGOS- 4654". We thank C. Antoniewski, P. Hollyoak, T. Hall and W. Hamitou for their contribution to some experiments and data analysis; N. Da Cruz and B. Leveille Nizerolle for technical assistance; as well as members of the Bardin team, N. Servant, J. Waterfall, D. Bourc'his, M. Greenberg and P.-A. Defossez for discussions and comments on the manuscript. This work was supported by grants from Fondation pour la Recherche Médicale (A.B., DEQ20160334928), as well as funding from the program "Investissements d'Avenir" launched by the French Government and implemented by ANR, ANR SoMuSeq-STEM (A.B), Labex DEEP (ANR-11-LBX-0044), IDEX PSL (ANR-10-IDEX-0001-02 PSL) and ICGex STEM-SOM-GERM (A.B. and K.S). Salary support of K.S. is from Inserm; A.J.B by CNRS; N.R is through a grant from Fondation ARC, and B.B by ENS Lyon.

\section{Author Contributions}

K.S., M.v.d.B. and A.J.B. designed the study and analyzed the data. M.v.d.B. developed software and performed all bioinformatic analysis. K.S. performed a majority of the experiments. B.B. collected samples for the Delta>nlsGFP background; M.S. obtained data for Supplemental Fig S4; and A.W. contributed to the long-read sequencing experiments. N.R. provided variant frequencies of neoplasia initiating events for Fig 3B. S.L. prepared samples for sequencing. K.S. and A.J.B. wrote the manuscript with contribution from M.v.d.B. and other authors.

The authors declare no competing interest. 


\section{References}

Ansaloni F, Scarpato M, Di Schiavi E, Gustincich S, Sanges R. 2019. Exploratory analysis of transposable elements expression in the C. elegans early embryo. BMC Bioinformatics 20: 484.

Antoniewski C. 2014. Computing siRNA and piRNA overlap signatures. Methods Mol Biol Clifton NJ 1173: $135-146$.

Bailey TL, Boden M, Buske FA, Frith M, Grant CE, Clementi L, Ren J, Li WW, Noble WS. 2009. MEME Suite: tools for motif discovery and searching. Nucleic Acids Res 37: W202-W208.

Baillie JK, Barnett MW, Upton KR, Gerhardt DJ, Richmond TA, De Sapio F, Brennan PM, Rizzu P, Smith S, Fell M, et al. 2011. Somatic retrotransposition alters the genetic landscape of the human brain. Nature 479: 534-537.

Berezikov E, Bucheton A, Busseau I. 2000. A search for reverse transcriptase-coding sequences reveals new non-LTR retrotransposons in the genome of Drosophila melanogaster. Genome Biol 1: research0012.1-12.15.

Bowen NJ, McDonald JF. 2001. Drosophila Euchromatic LTR Retrotransposons are Much Younger Than the Host Species in Which They Reside. Genome Res 11: 1527-1540.

Brennecke J, Aravin AA, Stark A, Dus M, Kellis M, Sachidanandam R, Hannon GJ. 2007. Discrete Small RNA-Generating Loci as Master Regulators of Transposon Activity in Drosophila. Cell 128: $1089-1103$.

Brennecke J, Malone CD, Aravin AA, Sachidanandam R, Stark A, Hannon GJ. 2008. An Epigenetic Role for Maternally Inherited piRNAs in Transposon Silencing. Science 322: 1387-1392.

Bucheton A, Paro R, Sang HM, Pelisson A, Finnegan DJ. 1984. The molecular basis of I-R hybrid dysgenesis in Drosophila melanogaster: identification, cloning, and properties of the I factor. Cell 38: 153-163.

Burns KH. 2017. Transposable elements in cancer. Nat Rev Cancer 17: 415-424.

Chambeyron S, Popkova A, Payen-Groschêne G, Brun C, Laouini D, Pelisson A, Bucheton A. 2008. piRNA-mediated nuclear accumulation of retrotransposon transcripts in the Drosophila female germline. Proc Natl Acad Sci 105: 14964-14969.

Chang Y-H, Keegan RM, Prazak L, Dubnau J. 2019. Cellular labeling of endogenous retrovirus replication (CLEVR) reveals de novo insertions of the gypsy retrotransposable element in cell culture and in both neurons and glial cells of aging fruit flies. PLOS Biol 17: e3000278.

Chen S, Zhou Y, Chen Y, Gu J. 2018. fastp: an ultra-fast all-in-one FASTQ preprocessor. Bioinformatics 34: i884-i890.

Chung N, Jonaid GM, Quinton S, Ross A, Sexton CE, Alberto A, Clymer C, Churchill D, Navarro Leija O, Han MV. 2019. Transcriptome analyses of tumor-adjacent somatic tissues reveal genes co-expressed with transposable elements. Mob DNA 10: 39.

Chung W-J, Okamura K, Martin R, Lai EC. 2008. Endogenous RNA Interference Provides a Somatic Defense against Drosophila Transposons. Curr Biol 18: 795-802.

Chuong EB, Elde NC, Feschotte C. 2017. Regulatory activities of transposable elements: from conflicts to benefits. Nat Rev Genet 18: 71-86. 
Cosby RL, Chang N-C, Feschotte C. 2019. Host-transposon interactions: conflict, cooperation, and cooption. Genes Dev 33: 1098-1116.

Coufal NG, Garcia-Perez JL, Peng GE, Yeo GW, Mu Y, Lovci MT, Morell M, O'Shea KS, Moran JV, Gage FH. 2009. L1 retrotransposition in human neural progenitor cells. Nature 460: 11271131.

Czech B, Malone CD, Zhou R, Stark A, Schlingeheyde C, Dus M, Perrimon N, Kellis M, Wohlschlegel JA, Sachidanandam R, et al. 2008. An endogenous small interfering RNA pathway in Drosophila. Nature 453: 798-802.

Dale RK, Pedersen BS, Quinlan AR. 2011. Pybedtools: a flexible Python library for manipulating genomic datasets and annotations. Bioinforma Oxf Engl 27: 3423-3424.

Deininger P, Morales ME, White TB, Baddoo M, Hedges DJ, Servant G, Srivastav S, Smither ME, Concha M, DeHaro DL, et al. 2017. A comprehensive approach to expression of L1 loci. Nucleic Acids Res 45: e31-e31.

Deniz Ö, Frost JM, Branco MR. 2019. Regulation of transposable elements by DNA modifications. Nat Rev Genet 20: 417-431.

Didion JP, Martin M, Collins FS. 2017. Atropos: specific, sensitive, and speedy trimming of sequencing reads. PeerJ 5: e3720.

Ding Y, Berrocal A, Morita T, Longden KD, Stern DL. 2016. Natural courtship song variation caused by an intronic retroelement in an ion channel gene. Nature 536: 329-332.

Doucet-O'Hare TT, Rodić N, Sharma R, Darbari I, Abril G, Choi JA, Ahn JY, Cheng Y, Anders RA, Burns KH, et al. 2015. LINE-1 expression and retrotransposition in Barrett's esophagus and esophageal carcinoma. Proc Natl Acad Sci 112: E4894-E4900.

Doucet-O'Hare TT, Sharmad R, Rodić N, Anders RA, Burns KH, Kazazian HH. 2016. Somatically acquired LINE-1 insertions in normal esophagus undergo clonal expansion in esophageal squamous cell carcinoma. Hum Mutat 37: 942-954.

Driver A, Lacey SF, Cullingford TE, Mitchelson A, O'Hare K. 1989. Structural analysis of Doc transposable elements associated with mutations at the white and suppressor of forked loci of Drosophila melanognster. Mol Gen Genet MGG 220: 49-52.

Dubnau J. 2018. The Retrotransposon storm and the dangers of a Collyer's genome. Curr Opin Genet Dev 49: 95-105.

Dunsmuir P, Brorein WJ, Simon MA, Rubin GM. 1980. Insertion of the Drosophila transposable element copia generates a 5 base pair duplication. Cell 21: 575-579.

Erwin JA, Marchetto MC, Gage FH. 2014. Mobile DNA elements in the generation of diversity and complexity in the brain. Nat Rev Neurosci 15: 497-506.

Evrony GD, Cai X, Lee E, Hills LB, Elhosary PC, Lehmann HS, Parker JJ, Atabay KD, Gilmore EC, Poduri A, et al. 2012. Single-Neuron Sequencing Analysis of L1 Retrotransposition and Somatic Mutation in the Human Brain. Cell 151: 483-496.

Evrony GD, Lee E, Mehta BK, Benjamini Y, Johnson RM, Cai X, Yang L, Haseley P, Lehmann HS, Park PJ, et al. 2015. Cell Lineage Analysis in Human Brain Using Endogenous Retroelements. Neuron 85: 49-59.

Evrony GD, Lee E, Park PJ, Walsh CA. 2016. Resolving rates of mutation in the brain using singleneuron genomics. eLife 5: e12966. 
Ewing AD, Gacita A, Wood LD, Ma F, Xing D, Kim M-S, Manda SS, Abril G, Pereira G, MakohonMoore A, et al. 2015. Widespread somatic L1 retrotransposition occurs early during gastrointestinal cancer evolution. Genome Res 25: 1536-1545.

Faulkner GJ, Garcia-Perez JL. 2017. L1 Mosaicism in Mammals: Extent, Effects, and Evolution. Trends Genet 33: 802-816.

Faulkner GJ, Kimura Y, Daub CO, Wani S, Plessy C, Irvine KM, Schroder K, Cloonan N, Steptoe AL, Lassmann T, et al. 2009. The regulated retrotransposon transcriptome of mammalian cells. Nat Genet 41: 563-571.

Feng Q, Moran JV, Kazazian HH, Boeke JD. 1996. Human L1 Retrotransposon Encodes a Conserved Endonuclease Required for Retrotransposition. Cell 87: 905-916.

Georgiev PG, Kiselev SL, Simonova OB, Gerasimova TI. 1990. A novel transposition system in Drosophila melanogaster depending on the Stalker mobile genetic element. EMBO J 9: 20372044.

Gerasimova TI, Matjunina LV, Mizrokhi LJ, Georgiev GP. 1985. Successive transposition explosions in Drosophila melanogaster and reverse transpositions of mobile dispersed genetic elements. EMBO J 4: 3773-3779.

Gervais L, van den Beek M, Josserand M, Sallé J, Stefanutti M, Perdigoto CN, Skorski P, Mazouni K, Marshall OJ, Brand AH, et al. 2019. Stem Cell Proliferation Is Kept in Check by the Chromatin Regulators Kismet/CHD7/CHD8 and Trr/MLL3/4. Dev Cell 49: 556-573.e6.

Ghildiyal M, Seitz H, Horwich MD, Li C, Du T, Lee S, Xu J, Kittler ELW, Zapp ML, Weng Z, et al. 2008. Endogenous siRNAs Derived from Transposons and mRNAs in Drosophila Somatic Cells. Science 320: 1077-1081.

Gogol-Döring A, Ammar I, Gupta S, Bunse M, Miskey C, Chen W, Uckert W, Schulz TF, Izsvák Z, Ivics Z. 2016. Genome-wide Profiling Reveals Remarkable Parallels Between Insertion Site Selection Properties of the MLV Retrovirus and the piggyBac Transposon in Primary Human CD4(+) T Cells. Mol Ther J Am Soc Gene Ther 24: 592-606.

Gong C, Maquat LE. 2011. IncRNAs transactivate STAU1-mediated mRNA decay by duplexing with 3' UTRs via Alu elements. Nature 470: 284-288.

Gunawardane LS, Saito K, Nishida KM, Miyoshi K, Kawamura Y, Nagami T, Siomi H, Siomi MC. 2007. A Slicer-Mediated Mechanism for Repeat-Associated siRNA 5’ End Formation in Drosophila. Science 315: 1587-1590.

Helman E, Lawrence MS, Stewart C, Sougnez C, Getz G, Meyerson M. 2014. Somatic retrotransposition in human cancer revealed by whole-genome and exome sequencing. Genome Res 24: 1053-1063.

Howard G, Eiges R, Gaudet F, Jaenisch R, Eden A. 2008. Activation and transposition of endogenous retroviral elements in hypomethylation induced tumors in mice. Oncogene 27: 404-408.

Iskow RC, McCabe MT, Mills RE, Torene S, Pittard WS, Neuwald AF, Van Meir EG, Vertino PM, Devine SE. 2010. Natural Mutagenesis of Human Genomes by Endogenous Retrotransposons. Cell 141: 1253-1261.

Jones BC, Wood JG, Chang C, Tam AD, Franklin MJ, Siegel ER, Helfand SL. 2016. A somatic piRNA pathway in the Drosophila fat body ensures metabolic homeostasis and normal lifespan. Nat Commun 7: 13856.

Kharchenko PV, Alekseyenko AA, Schwartz YB, Minoda A, Riddle NC, Ernst J, Sabo PJ, Larschan E, Gorchakov AA, Gu T, et al. 2011. Comprehensive analysis of the chromatin landscape in Drosophila melanogaster. Nature 471: 480-485. 
Kidwell MG, Kidwell JF, Sved JA. 1977. Hybrid Dysgenesis in DROSOPHILA MELANOGASTER: A Syndrome of Aberrant Traits Including Mutation, Sterility and Male Recombination. Genetics 86: 813-833.

Langmead B, Trapnell C, Pop M, Salzberg SL. 2009. Ultrafast and memory-efficient alignment of short DNA sequences to the human genome. Genome Biol 10: R25.

Lee E, Iskow R, Yang L, Gokcumen O, Haseley P, Luquette LJ, Lohr JG, Harris CC, Ding L, Wilson RK, et al. 2012. Landscape of Somatic Retrotransposition in Human Cancers. Science 337: 967-971.

Li H. 2018. Minimap2: pairwise alignment for nucleotide sequences. Bioinformatics 34: 3094-3100.

Li W, Prazak L, Chatterjee N, Grüninger S, Krug L, Theodorou D, Dubnau J. 2013. Activation of transposable elements during aging and neuronal decline in Drosophila. Nat Neurosci 16: 529-531.

Linheiro RS, Bergman CM. 2012. Whole Genome Resequencing Reveals Natural Target Site Preferences of Transposable Elements in Drosophila melanogaster. PLOS ONE 7: e30008.

Love MI, Huber W, Anders S. 2014. Moderated estimation of fold change and dispersion for RNA-seq data with DESeq2. Genome Biol 15: 550.

Macia A, Widmann TJ, Heras SR, Ayllon V, Sanchez L, Benkaddour-Boumzaouad M, Muñoz-Lopez M, Rubio A, Amador-Cubero S, Blanco-Jimenez E, et al. 2017. Engineered LINE-1 retrotransposition in nondividing human neurons. Genome Res 27: 335-348.

Marshall OJ, Brand AH. 2017. Chromatin state changes during neural development revealed by in vivo cell-type specific profiling. Nat Commun 8: 2271.

Mateo L, Ullastres A, González J. 2014. A Transposable Element Insertion Confers Xenobiotic Resistance in Drosophila ed. C. Feschotte. PLoS Genet 10: e1004560.

Micchelli CA, Perrimon N. 2006. Evidence that stem cells reside in the adult Drosophila midgut epithelium. Nature 439: 475-479.

Mietz JA, Fewell JW, Kuff EL. 1992. Selective activation of a discrete family of endogenous proviral elements in normal BALB/c lymphocytes. Mol Cell Biol 12: 220-228.

Miki Y, Nishisho I, Horii A, Miyoshi Y, Utsunomiya J, Kinzler KW, Vogelstein B, Nakamura Y. 1992. Disruption of the APC gene by a retrotransposal insertion of L1 sequence in a colon cancer. Cancer Res 52: 643-645.

Molaro A, Malik HS. 2016. Hide and seek: how chromatin-based pathways silence retroelements in the mammalian germline. Curr Opin Genet Dev 37: 51-58.

Muotri AR, Chu VT, Marchetto MCN, Deng W, Moran JV, Gage FH. 2005. Somatic mosaicism in neuronal precursor cells mediated by L1 retrotransposition. Nature 435: 903-910.

Nègre N, Brown CD, Ma L, Bristow CA, Miller SW, Wagner U, Kheradpour P, Eaton ML, Loriaux P, Sealfon R, et al. 2011. A Cis-Regulatory Map of the Drosophila Genome. Nature 471: 527531.

Ohlstein B, Spradling A. 2006. The adult Drosophila posterior midgut is maintained by pluripotent stem cells. Nature 439: 470-474.

Ong-Abdullah M, Ordway JM, Jiang N, Ooi S-E, Kok S-Y, Sarpan N, Azimi N, Hashim AT, Ishak Z, Rosli SK, et al. 2015. Loss of Karma transposon methylation underlies the mantled somaclonal variant of oil palm. Nature 525: 533-537. 
Patel PH, Dutta D, Edgar BA. 2015. Niche appropriation by Drosophila intestinal stem cell tumours. Nat Cell Biol 17: 1182-1192.

Paterson AL, Weaver JMJ, Eldridge MD, Tavaré S, Fitzgerald RC, Edwards PAW. 2015. Mobile element insertions are frequent in oesophageal adenocarcinomas and can mislead paired-end sequencing analysis. BMC Genomics 16.

https://www.ncbi.nlm.nih.gov/pmc/articles/PMC4498532/ (Accessed December 17, 2019).

Patro R, Duggal G, Love MI, Irizarry RA, Kingsford C. 2017. Salmon provides fast and bias-aware quantification of transcript expression. Nat Methods 14: 417-419.

Pehrsson EC, Choudhary MNK, Sundaram V, Wang T. 2019. The epigenomic landscape of transposable elements across normal human development and anatomy. Nat Commun 10: 116.

Pélisson A. 1981. The I--R system of hybrid dysgenesis in Drosophila melanogaster: are I factor insertions responsible for the mutator effect of the I--R interaction? Mol Gen Genet MGG 183: 123-129.

Perrat PN, DasGupta S, Wang J, Theurkauf W, Weng Z, Rosbash M, Waddell S. 2013. TranspositionDriven Genomic Heterogeneity in the Drosophila Brain. Science 340: 91-95.

Philippe C, Vargas-Landin DB, Doucet AJ, van Essen D, Vera-Otarola J, Kuciak M, Corbin A, Nigumann P, Cristofari G. 2016. Activation of individual L1 retrotransposon instances is restricted to cell-type dependent permissive loci ed. K. Burns. eLife 5: e13926.

Quinlan AR, Hall IM. 2010. BEDTools: a flexible suite of utilities for comparing genomic features. Bioinforma Oxf Engl 26: 841-842.

Riddle NC, Minoda A, Kharchenko PV, Alekseyenko AA, Schwartz YB, Tolstorukov MY, Gorchakov AA, Jaffe JD, Kennedy C, Linder-Basso D, et al. 2011. Plasticity in patterns of histone modifications and chromosomal proteins in Drosophila heterochromatin. Genome Res 21: 147.

Rodić N, Steranka JP, Makohon-Moore A, Moyer A, Shen P, Sharma R, Kohutek ZA, Huang CR, Ahn D, Mita P, et al. 2015. Retrotransposon insertions in the clonal evolution of pancreatic ductal adenocarcinoma. Nat Med 21: 1060-1064.

Rodriguez-Martin B, Alvarez EG, Baez-Ortega A, Zamora J, Supek F, Demeulemeester J, Santamarina M, Ju YS, Temes J, Garcia-Souto D, et al. 2020. Pan-cancer analysis of whole genomes identifies driver rearrangements promoted by LINE-1 retrotransposition. Nat Genet 52: 306-319.

Rubin GM, Kidwell MG, Bingham PM. 1982. The molecular basis of P-M hybrid dysgenesis: the nature of induced mutations. Cell 29: 987-994.

Sanchez-Luque FJ, Kempen M-JHC, Gerdes P, Vargas-Landin DB, Richardson SR, Troskie R-L, Jesuadian JS, Cheetham SW, Carreira PE, Salvador-Palomeque C, et al. 2019. LINE-1 Evasion of Epigenetic Repression in Humans. Mol Cell 75: 590-604.e12.

Sang HM, Pélisson A, Bucheton A, Finnegan DJ. 1984. Molecular lesions associated with white gene mutations induced by I-R hybrid dysgenesis in Drosophila melanogaster. EMBO J 3: 30793085 .

Schauer SN, Carreira PE, Shukla R, Gerhardt DJ, Gerdes P, Sanchez-Luque FJ, Nicoli P, Kindlova M, Ghisletti S, Santos AD, et al. 2018. L1 retrotransposition is a common feature of mammalian hepatocarcinogenesis. Genome Res 28: 639-653. 
Scott EC, Gardner EJ, Masood A, Chuang NT, Vertino PM, Devine SE. 2016. A hot L1 retrotransposon evades somatic repression and initiates human colorectal cancer. Genome Res 26: 745-755.

Shen S, Lin L, Cai JJ, Jiang P, Kenkel EJ, Stroik MR, Sato S, Davidson BL, Xing Y. 2011. Widespread establishment and regulatory impact of Alu exons in human genes. Proc Natl Acad Sci 108: 2837-2842.

Shi Y, Lan F, Matson C, Mulligan P, Whetstine JR, Cole PA, Casero RA, Shi Y. 2004. Histone Demethylation Mediated by the Nuclear Amine Oxidase Homolog LSD1. Cell 119: 941-953.

Shukla R, Upton KR, Muñoz-Lopez M, Gerhardt DJ, Fisher ME, Nguyen T, Brennan PM, Baillie JK, Collino A, Ghisletti S, et al. 2013. Endogenous Retrotransposition Activates Oncogenic Pathways in Hepatocellular Carcinoma. Cell 153: 101-111.

Siudeja K, Bardin AJ. 2017. Somatic recombination in adult tissues: What is there to learn? Fly (Austin) 11: 121-128.

Siudeja K, Nassari S, Gervais L, Skorski P, Lameiras S, Stolfa D, Zande M, Bernard V, Frio TR, Bardin AJ. 2015. Frequent Somatic Mutation in Adult Intestinal Stem Cells Drives Neoplasia and Genetic Mosaicism during Aging. Cell Stem Cell 17: 663-674.

Solyom S, Ewing AD, Rahrmann EP, Doucet T, Nelson HH, Burns MB, Harris RS, Sigmon DF, Casella A, Erlanger B, et al. 2012. Extensive somatic L1 retrotransposition in colorectal tumors. Genome Res 22: 2328-2338.

Sousa-Victor P, Ayyaz A, Hayashi R, Qi Y, Madden DT, Lunyak VV, Jasper H. 2017. Piwi Is Required to Limit Exhaustion of Aging Somatic Stem Cells. Cell Rep 20: 2527-2537.

Stefano LD, Ji J-Y, Moon N-S, Herr A, Dyson N. 2007. Mutation of Drosophila Lsd1 Disrupts H3-K4 Methylation, Resulting in Tissue-Specific Defects during Development. Curr Biol 17: 808-812.

Sultana T, van Essen D, Siol O, Bailly-Bechet M, Philippe C, Zine El Aabidine A, Pioger L, Nigumann P, Saccani S, Andrau J-C, et al. 2019. The Landscape of L1 Retrotransposons in the Human Genome Is Shaped by Pre-insertion Sequence Biases and Post-insertion Selection. Mol Cell 74: 555-570.e7.

Sultana T, Zamborlini A, Cristofari G, Lesage P. 2017. Integration site selection by retroviruses and transposable elements in eukaryotes. Nat Rev Genet 18: 292-308.

Tang Z, Steranka JP, Ma S, Grivainis M, Rodić N, Huang CRL, Shih I-M, Wang T-L, Boeke JD, Fenyö D, et al. 2017. Human transposon insertion profiling: Analysis, visualization and identification of somatic LINE-1 insertions in ovarian cancer. Proc Natl Acad Sci 114: E733-E740.

The modENCODE Consortium, Roy S, Ernst J, Kharchenko PV, Kheradpour P, Negre N, Eaton ML, Landolin JM, Bristow CA, Ma L, et al. 2010. Identification of Functional Elements and Regulatory Circuits by Drosophila modENCODE. Science 330: 1787-1797.

Treiber CD, Waddell S. 2017. Resolving the prevalence of somatic transposition in Drosophila. eLife 6: e28297.

Truett MA, Jones RS, Potter SS. 1981. Unusual structure of the FB family of transposable elements in Drosophila. Cell 24: 753-763.

Tubio JMC, Li Y, Ju YS, Martincorena I, Cooke SL, Tojo M, Gundem G, Pipinikas CP, Zamora J, Raine K, et al. 2014. Extensive transduction of nonrepetitive DNA mediated by L1 retrotransposition in cancer genomes. Science 345: 1251343-1251343. 
Upton KR, Gerhardt DJ, Jesuadian JS, Richardson SR, Sánchez-Luque FJ, Bodea GO, Ewing AD, Salvador-Palomeque C, van der Knaap MS, Brennan PM, et al. 2015. Ubiquitous L1 Mosaicism in Hippocampal Neurons. Cell 161: 228-239.

Van't Hof AE, Campagne P, Rigden DJ, Yung CJ, Lingley J, Quail MA, Hall N, Darby AC, Saccheri IJ. 2016. The industrial melanism mutation in British peppered moths is a transposable element. Nature 534: 102-105.

Wang L, Zeng X, Ryoo HD, Jasper H. 2014. Integration of UPRER and Oxidative Stress Signaling in the Control of Intestinal Stem Cell Proliferation ed. N. Perrimon. PLoS Genet 10: e1004568.

Whalen JH, Grigliatti TA. 1998. Molecular characterization of a retrotransposon in Drosophila melanogaster, nomad, and its relationship to other retrovirus-like mobile elements. Mol Gen Genet MGG 260: 401-409.

Wood JG, Jones BC, Jiang N, Chang C, Hosier S, Wickremesinghe P, Garcia M, Hartnett DA, Burhenn L, Neretti N, et al. 2016. Chromatin-modifying genetic interventions suppress ageassociated transposable element activation and extend life span in Drosophila. Proc Natl Acad Sci 113: 11277-11282. 


\section{Siudeja_Fig1}

A

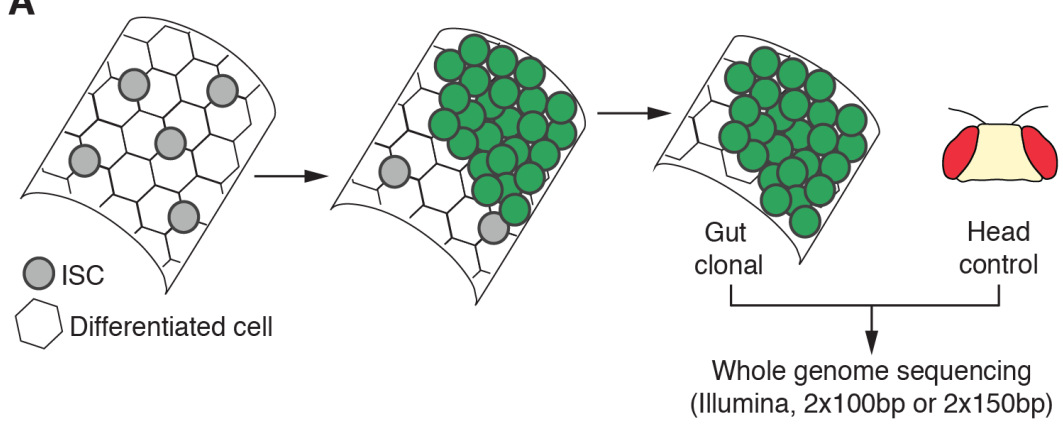

C

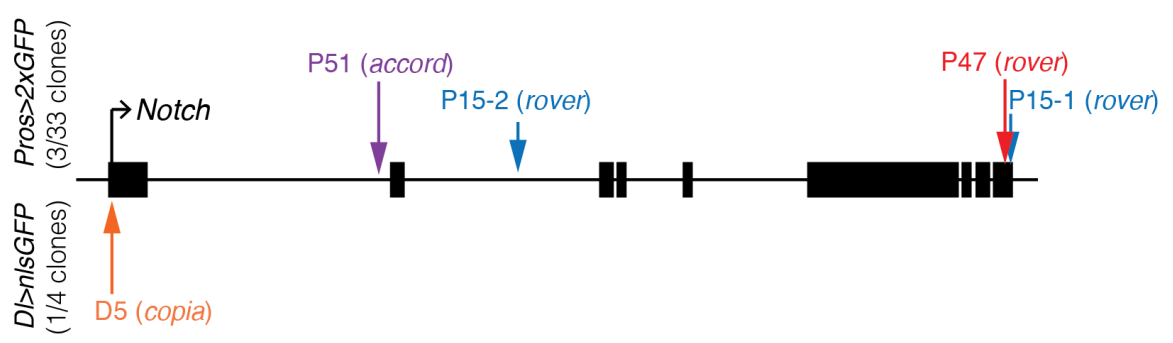

D

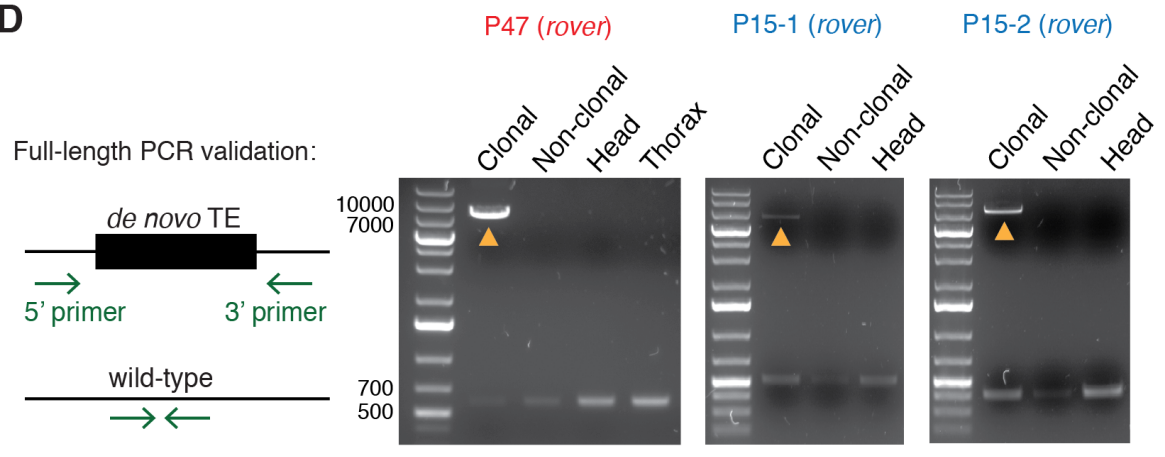

E

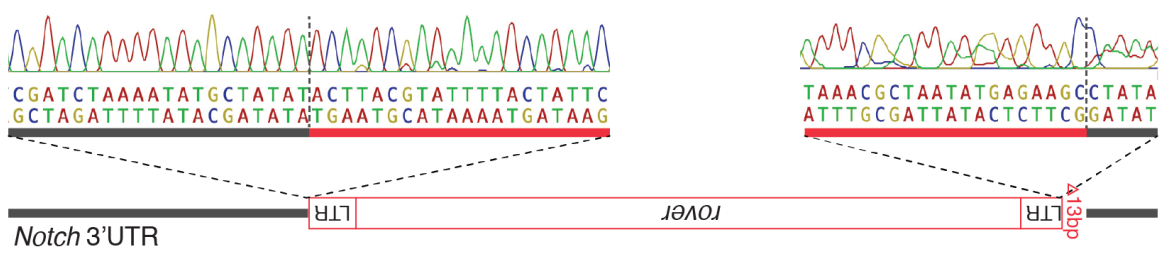

B

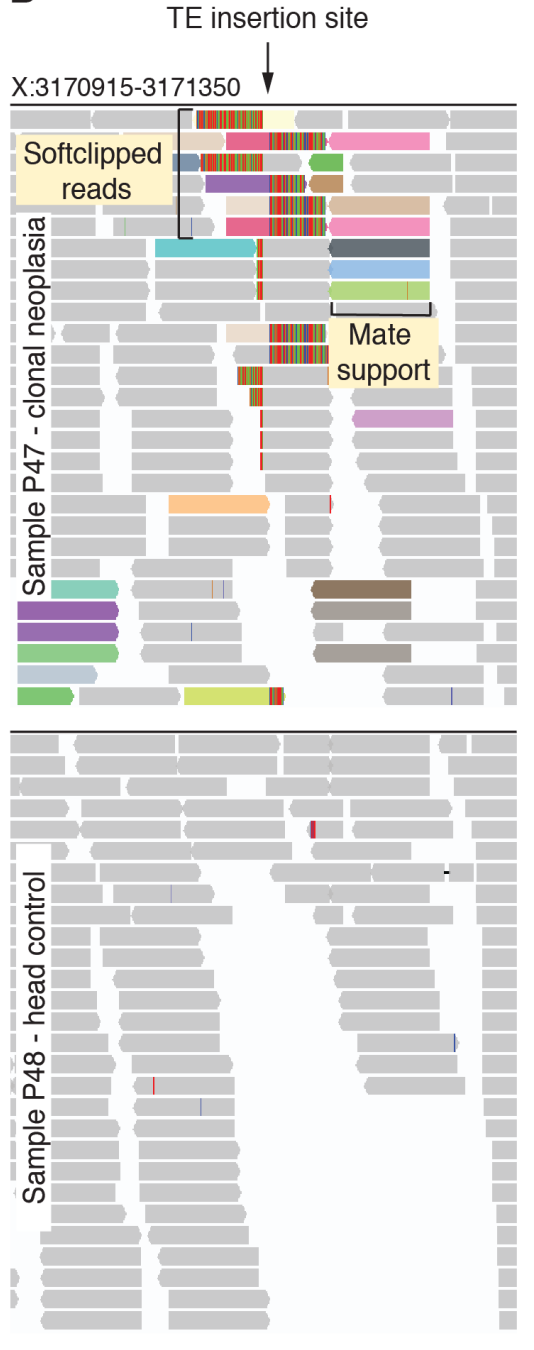

Notch 3'UTR

Figure 1. Somatic TE insertions in Notch in spontaneous male neoplasia

(A) The fly intestine is maintained by the Intestinal Stem Cells (ISCs). Inactivation of Notch in a stem cell (in green) leads to a clonal expansion of the mutant cell and neoplasia. The neoplastic gut region was microdissected together with the head of the same fly. DNA isolated from both tissues was subjected to whole-genome paired-end sequencing. (B) An Integrative Genomics Viewer (IGV) screenshot of the Notch de novo TE insertion site from sample P47 (clonal neoplasia) and its head control, sample P48. Bars represent sequencing reads. Reads supporting the TE insertion are colored according to homology to a specific TE insertion sequence. Multiple colors at a putative insert site frequently indicate homology to different insertions of the same TE family. Two types of supporting reads can be seen: soft-clipped reads - spanning the insertion site and mapping partially to the reference genome and partially to the TE, and mate pair support reads - flanking the insertion site and mapping 
to the reference genome but with mates (not seen) mapping to the TE. (C) The Notch locus and the identified somatic TE insertion sites indicated with vertical arrows. Black bars represent exons. Insertions in Notch were identified in 3 out of 33 clonal samples from the Pros>2xGFP genetic background and in 1 out of 4 D/>ns/GFP samples. (D) PCR validation of 3 somatic, neoplasia-specific insertions of rover elements identified in samples R47 and P15. Primers were designed to target regions flanking the insertion sites. Yellow arrowheads indicate PCR products containing around $8 \mathrm{~kb}$ insertion amplified in the clonal DNA but not in the neighboring gut tissue (non-clonal), head or thorax for the same fly. Short wild-type amplicon was detectable in all samples. Thorax DNA sample was not available for sample P15. (E) Sanger sequencing of the TE insertion breakpoints in the 3'UTR of Notch from sample P47. The rover LTR element was inserted in a reverse orientation to Notch. The 5' LTR sequence was truncated by $13 \mathrm{bp}$. Vertical dashed lines indicate insertion breakpoints. LTR - long terminal repeat 


\section{Siudeja_Fig2}

A

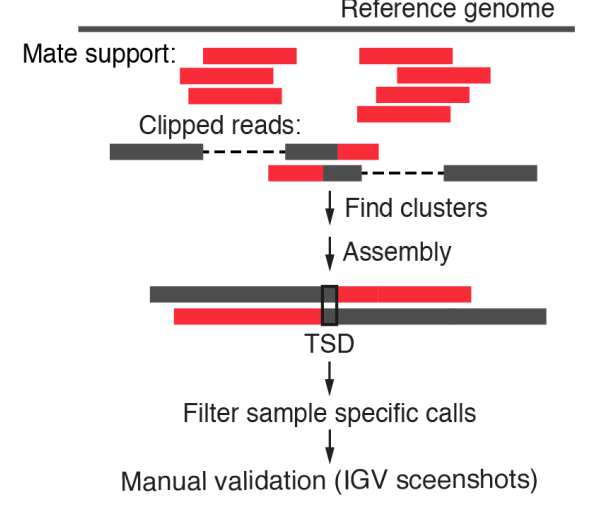

C

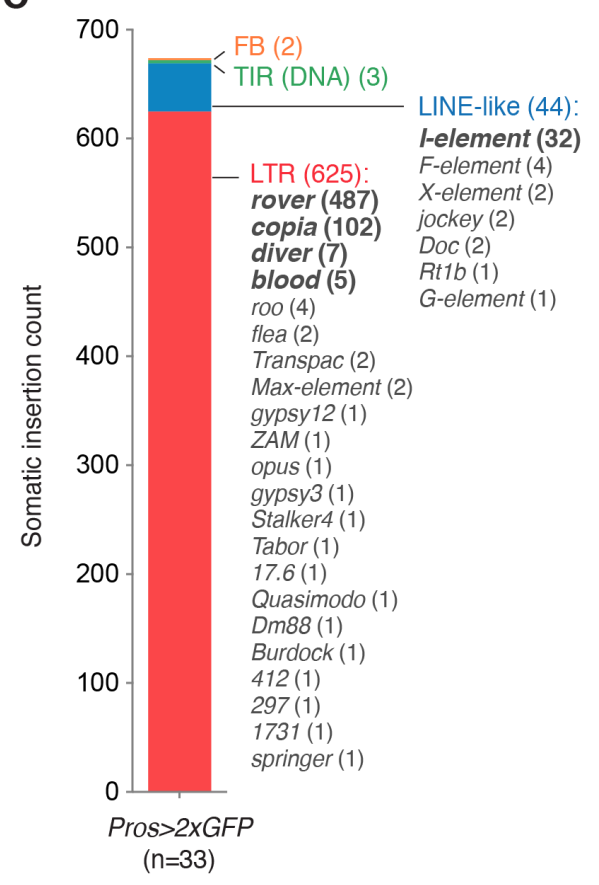

D

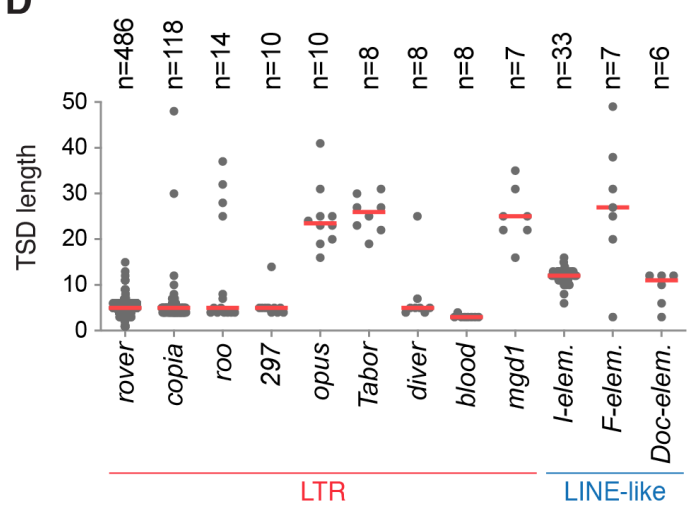

B
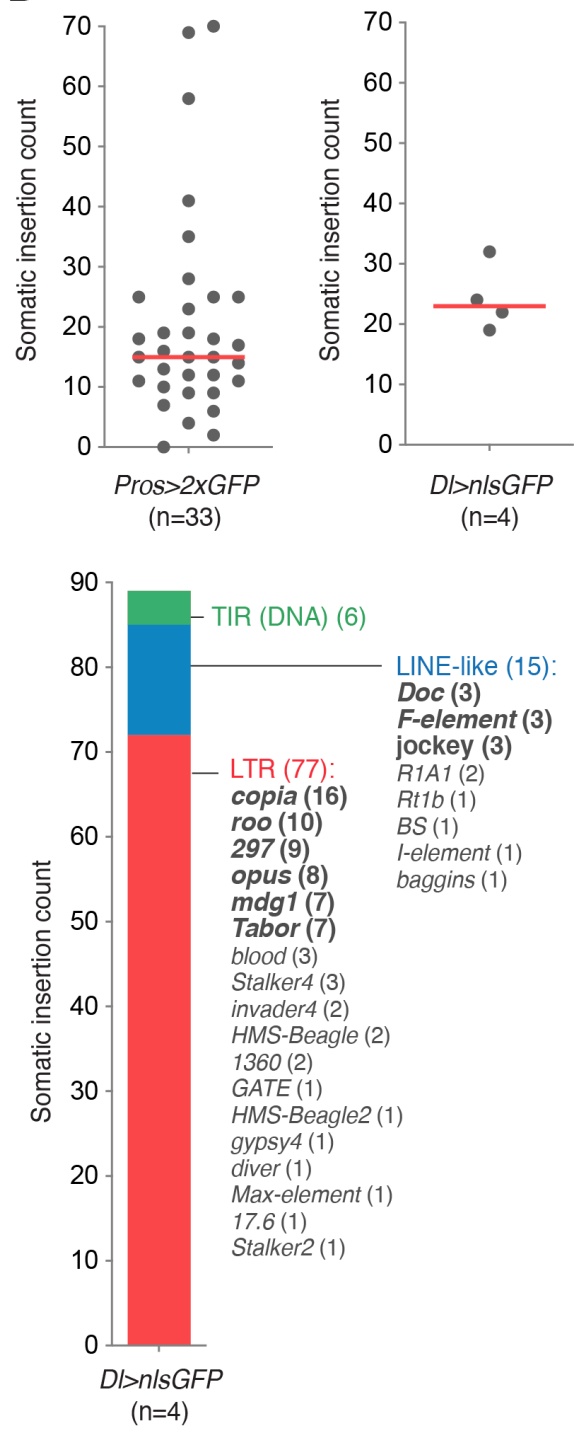

E

rover - target motif $(p=1.4 e-716)$

\section{Figure 2. Retrotransposition occurs genome-wide in the fly midgut}

(A) The bioinformatic pipeline used to identify somatic TE insertions in short-read sequencing datasets. Two types of supporting reads are identified genome-wide: mate support reads, where one of the paired-end reads is mapped to the reference genome, while the other mate (not shown) is associated with a TE, and clipped reads, which span the insertion site and map partially to the reference genome 
and partially to a TE. Isolated reads were then clustered and assembled to map individual insertion sites. Only insertions with a valid target site duplication (TSD) were retained, sample specific calls were filtered and manual validation of each candidate insertion was performed on IGV. (B) The frequency of gut specific somatic insertion sites in the Pros>2XGFP and Delta>nlsGFP genetic backgrounds. Red lines represent median values. (C) The distribution of TE classes active in the two genetic backgrounds studied. TEs were categorized in four main classes: LTR - long terminal repeat retrotransposons, LINElike - non-LTR retrotransposons, TIR - terminal inverted repeat DNA transposons, and FB - fodlback element (D) TSD length distribution for somatic insertions of most frequent TE families. Insertions from both genotypes were pooled. Red lines represent median values. (E) The target site motif at rover-LTR insertions sites recovered from the clonal gut samples. 


\section{Siudeja_Fig3}

A

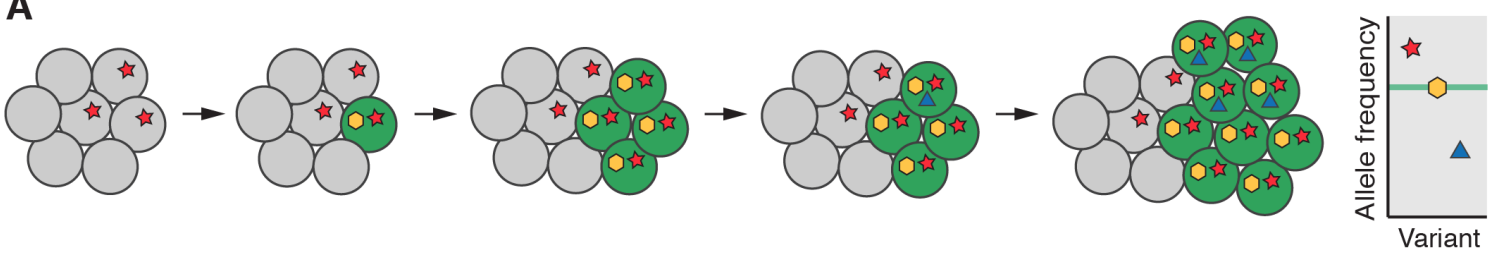

\section{B}
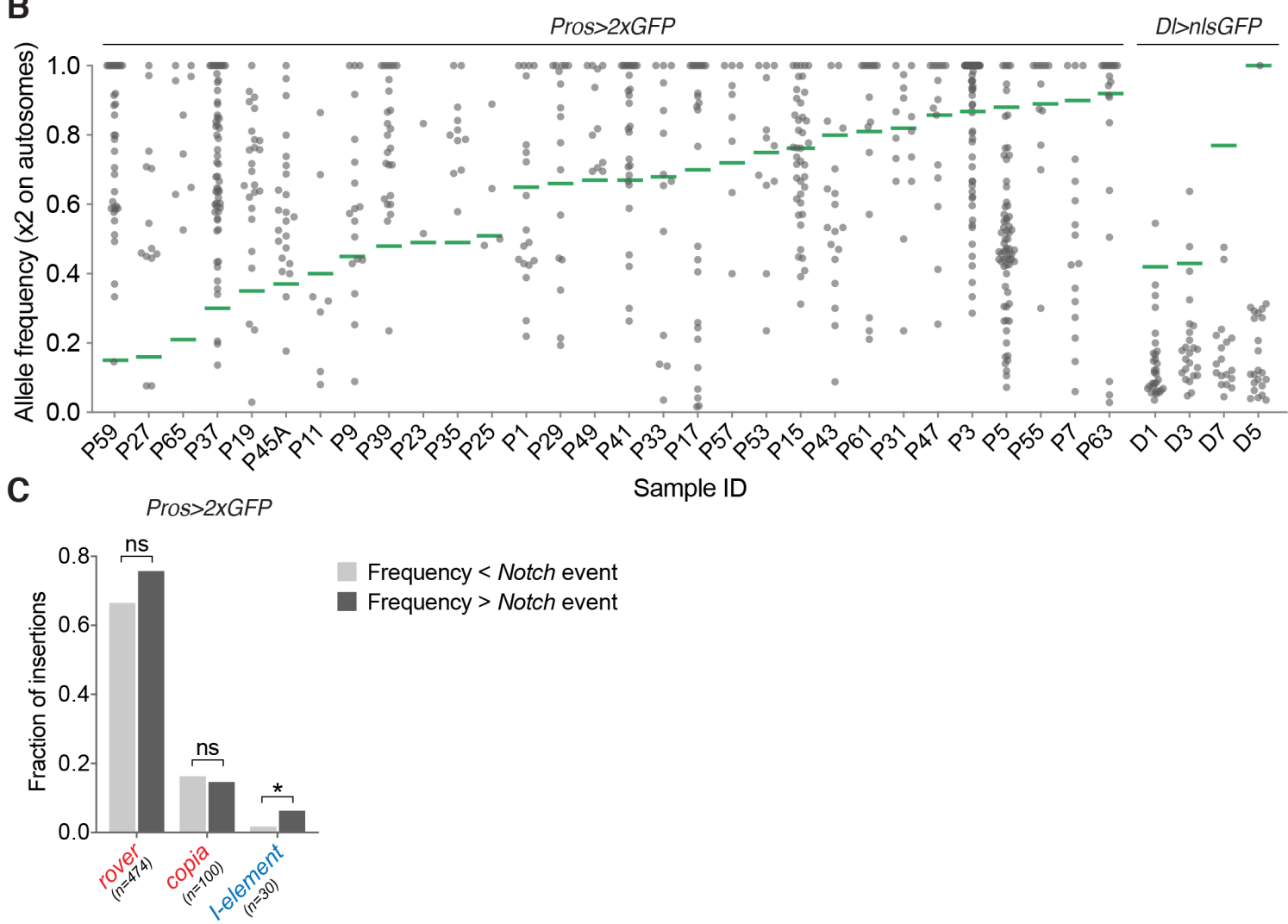

Frequency $<$ Notch event

Frequency $>$ Notch event

\section{Figure 3. Somatic retrotransposition occurs before and after the clonal expansion}

(A) A somatic insertion may arise in a normal tissue and be present in a fraction of cells of the tissue (red star). If a second somatic event (yellow) inactivates the Notch pathway, the mutant cell (indicated in green) will initiate the clonal expansion amplifying somatic variants already present in its genome. Finally, any insertion that occurs after the clonal expansion (blue triangle) will be present in a subset of neoplastic cells. The graph represents theoretical allele frequencies of somatic variants obtained from the sequencing of clonal tissue samples. The allele frequency of a Notch inactivating event, marking the onset of neoplasia is represented with a green horizontal line. A somatic insertion with allele frequency higher than the Notch inactivating event was likely present in the tissue before the clonal expansion. In contrast, an insertion with allele frequency lower than the Notch inactivating event, likely occurred after the initiation of neoplasia and is thus subclonal. (B) The calculated allele frequencies for all somatic TE insertions identified in neoplastic samples (Pros>2GFP and DI>n/GFP genetic backgrounds). The onset of neoplasia is represented with a green horizontal line. Three samples (P45B, P51 and P21), where timing of the neoplasia onset could not be unambiguously estimated, were excluded from this analysis. (C) The distribution of somatic insertions with frequencies higher or lower than Notch-inactivating 
bioRxiv preprint doi: https://doi.org/10.1101/2020.07.10.166629; this version posted July 10, 2020. The copyright holder for this preprint (which was not certified by peer review) is the author/funder. All rights reserved. No reuse allowed without permission.

mutations for the most represented LTR-elements (rover and copia) and LINE-like l-elements. ns- not significant, *- $p<0.01$ (Fisher's exact test, two-tailed), $n=$ number of insertions 


\section{Siudeja_Fig4}

A

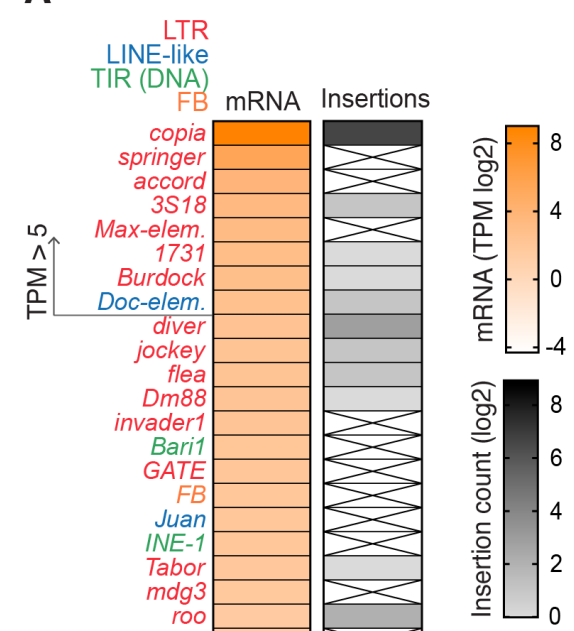

B
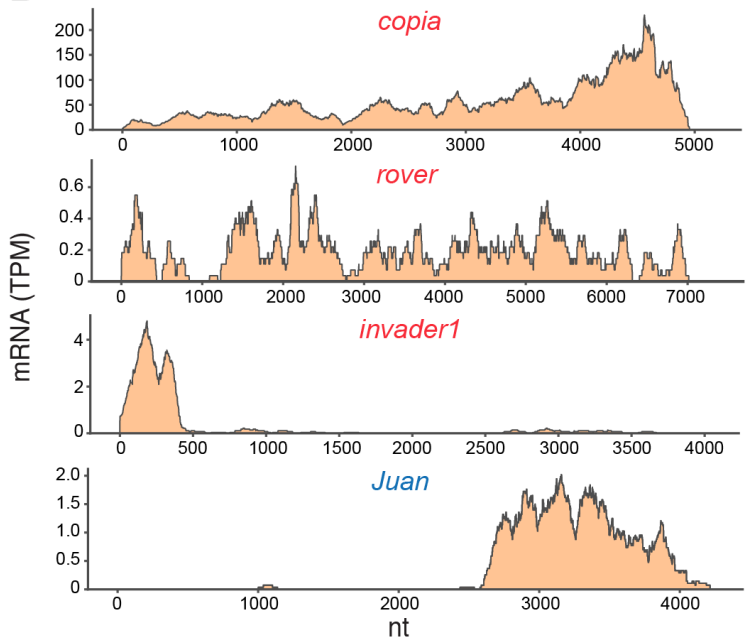

D
C

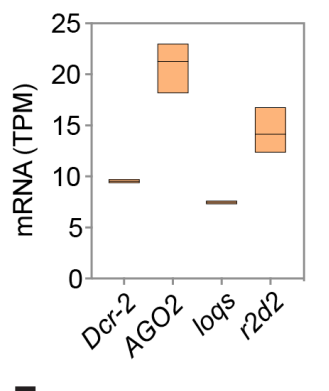

E

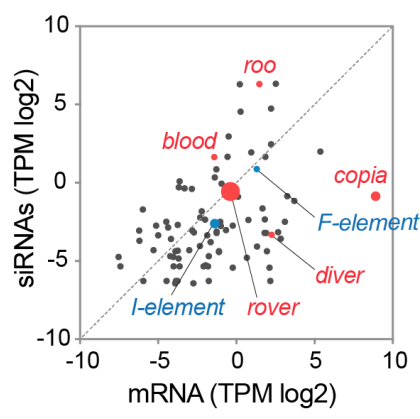

D Pros $>2 X G F P-\mathrm{R} 1 \quad$ Pros $>2 X G F P-\mathrm{R} 2$

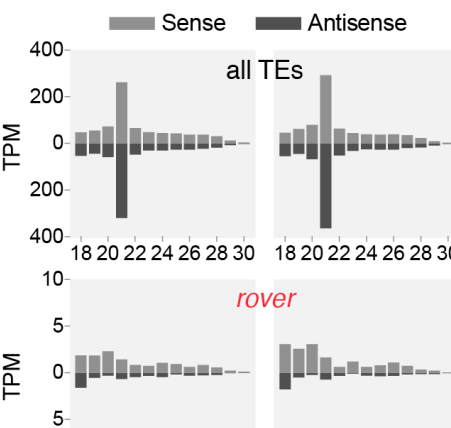

$18202224262830 \quad 18202224262830$

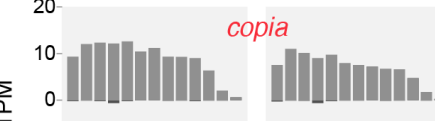

$\stackrel{10}{F}$

10

20-18202224262830 18202224262830

10-element

$\sum_{\substack{1 \\ \vdash}} \begin{aligned} & 5 \\ & 5\end{aligned}$

$10 \quad 18202224262830 \quad 18202224262830$

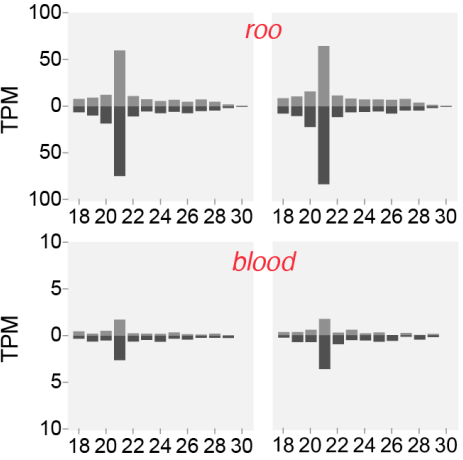
Read length (nt)

Figure 4. TE expression and siRNA pathway activity in the fly midgut

(A) Heatmaps representing normalized TE expression levels (in log2(TPM), transcripts per million) and mobility (log2(insertion counts)) in Pros $>2 x G F P$ midguts. TEs with TPM values below $0.05(\log 2(T P M)<$ 
-4.3) are not depicted. Crossed out cells represent no somatic insertions of that family identified. (B) Normalized read coverage over the full-length canonical sequence of selected TE families. (C) Normalized expression levels of siRNA pathway genes. Bars represent the minimum, the maximum and the mean from three biological replicates. (D) The size distribution of sense and antisense reads from gut small RNA fractions mapping to all TEs (upper panel) or selected TE families mobilizing in the gut. R1 and R2 are two biological replicates. (E) Scatter plot of normalized transcript (mRNA) levels and antisense, 21nt siRNA levels for all TE families. Transposons generating somatic insertions are highlighted in red (LTR-elements) or blue (LINE-like), with a symbol size reflecting the somatic insertion counts. 


\section{Siudeja_Fig5}

A
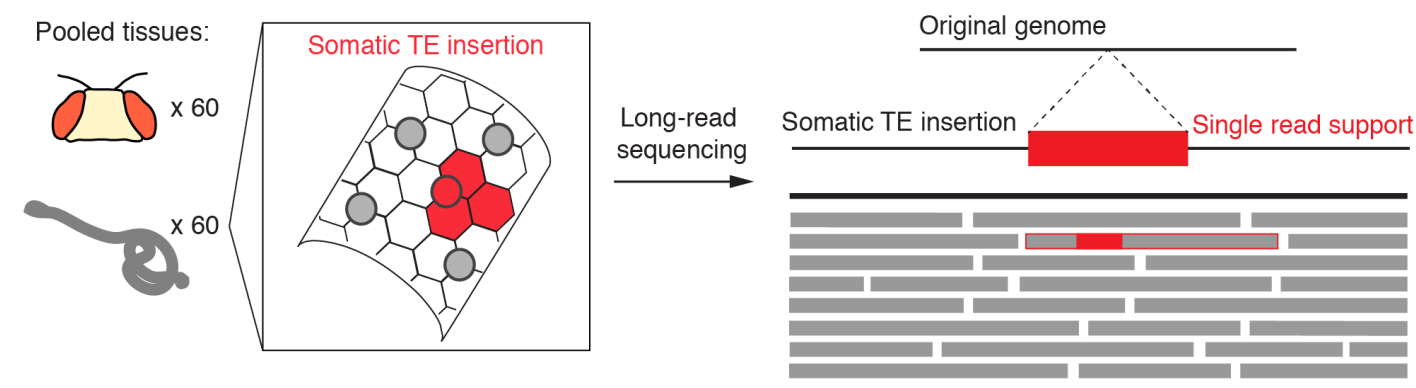

B

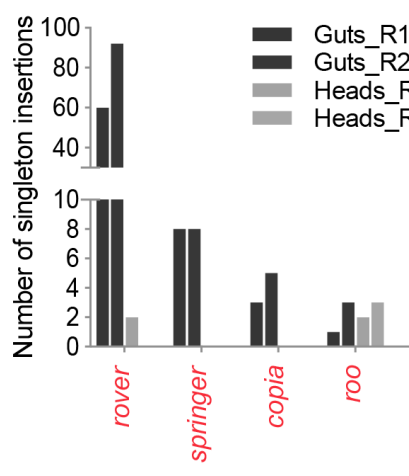

C rover - target motif $(p=5.8 e-287)$

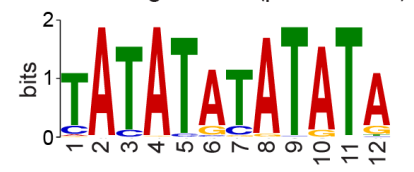

Figure 5. Long-read sequencing implies tissue specific transposon mobility

(A) Genomic DNA was isolated from pools of 60 guts or 60 heads dissected from the same individuals and subjected to ONT long-read sequencing. If a somatic insertion occurs in a tissue and does not undergo clonal expansion, it will be present in a small fraction of cells within the pool sequenced. Longread sequencing allows to identify putative somatic integrations as rare TE inserts, fully contained in a single continuous sequencing read and generating a valid TSD. (B) TE families with tissue-specific, singleton TSD-bearing insertions detected in pooled gut or head DNA. R1 and R2 are two biological replicates. (C) The target site motif at gut-specific rover singleton insertion sites identified with the longread sequencing. 


\section{Siudeja_Fig6}

A A

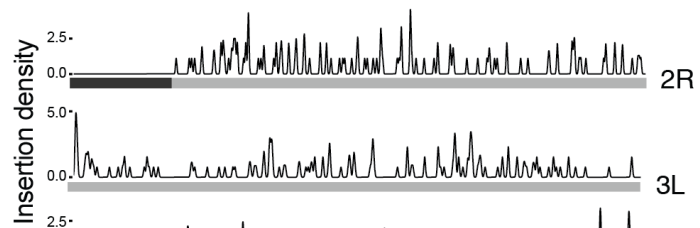

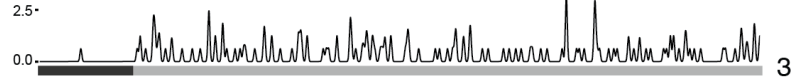

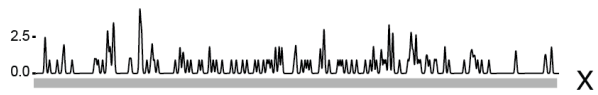

B

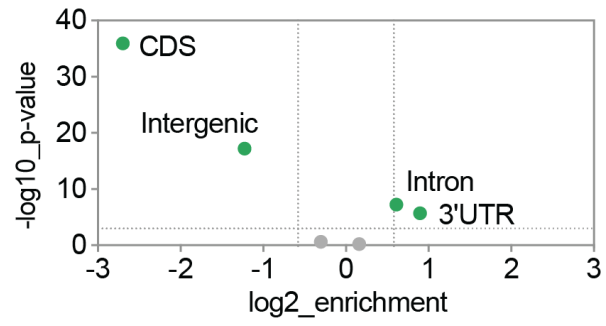

D

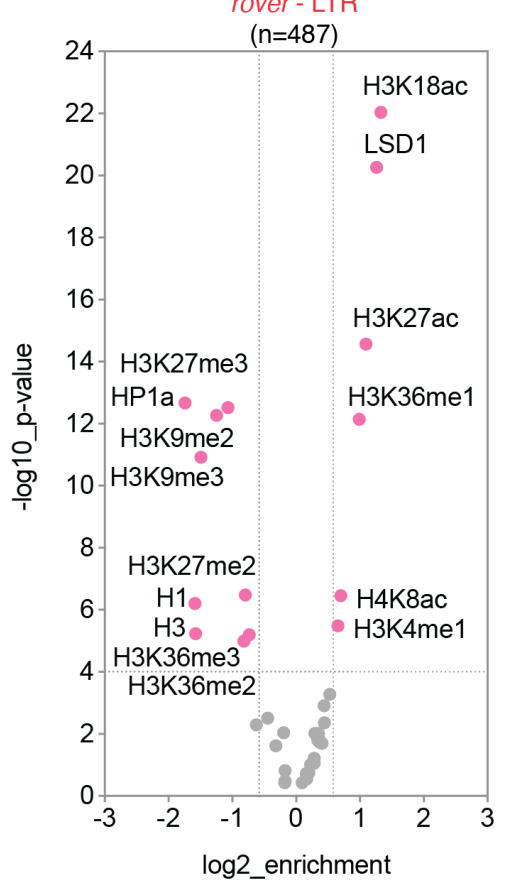

$\mathbf{E}$

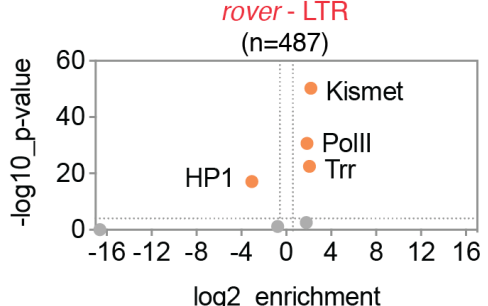

$\log 2$ enrichment
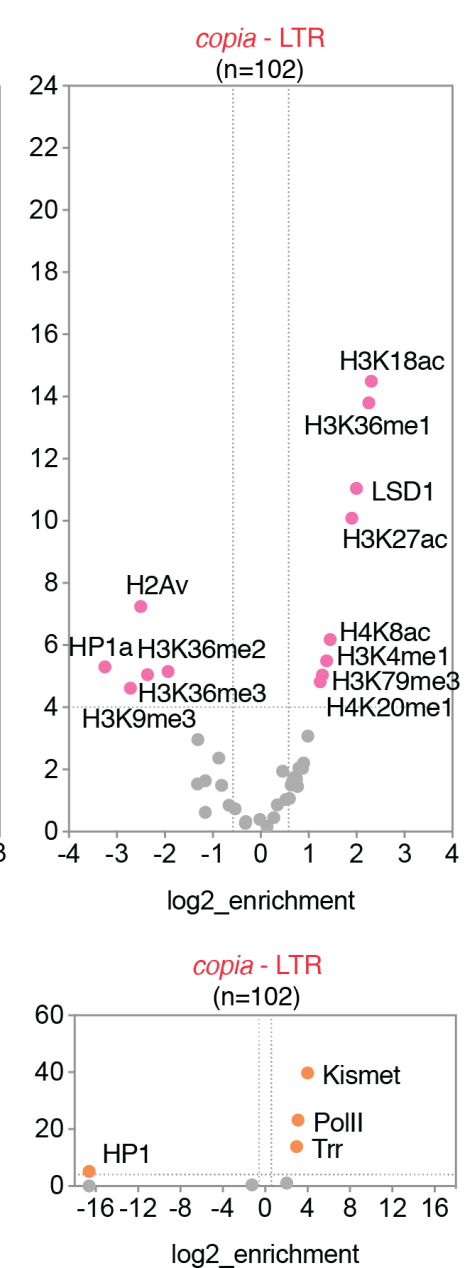

C

\begin{tabular}{|lll|}
\hline Hits & Gene & Function \\
\hline Clonal samples (short-read seq): \\
5 & pointed & EGFR pathway \\
3 & puckered & JNK pathway \\
$3^{*}$ & Notch & Notch pathway \\
2 & scribble & Epithelial polarity \\
1 & kuzbanian & Notch pathway \\
1 & neuralized & Notch pathway \\
1 & Stat92E & JAK/STAT pathway \\
1 & frizzled & Wnt pathway \\
1 & Nipped-A & ISC proliferation \\
1 & kismet & Chromatin organization \\
1 & Osa & Chromatin organization \\
1 & Atg8a & Autophagy \\
1 & robo1 & Lineage specification \\
Pooled guts (long-read seq): \\
1 & EGFR & EGFR pathway \\
1 & InR & Insulin pathway \\
1 & Myc & ISC proliferation \\
1 & Pvf1 & VEGF pathway \\
1 & Pvf2 & VEGF pathway \\
1 & Pvf3 & VEGF pathway \\
1 & Piezo & Lineage specification \\
1 & pros & Lineage specification \\
* Insertions with a valid TSD. \\
1 additional Notch insertion without TSD is reported \\
in Fig1 & & \\
\multicolumn{4}{c}{} & &
\end{tabular}

l-element - LINE-like

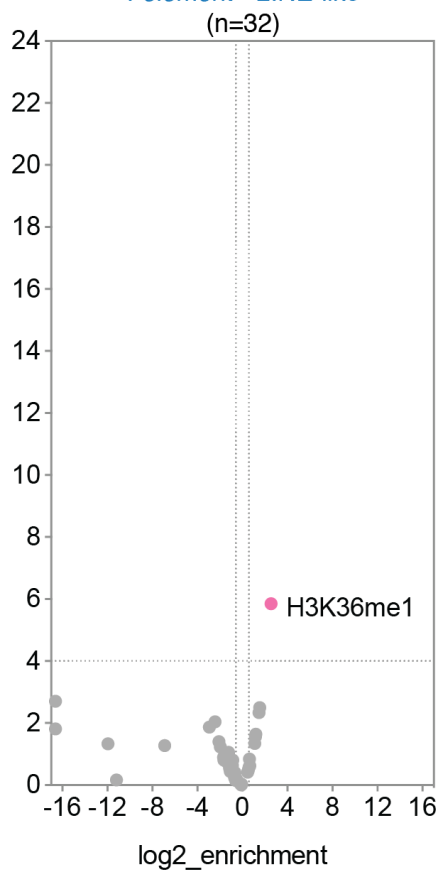

I-element - LINE-like

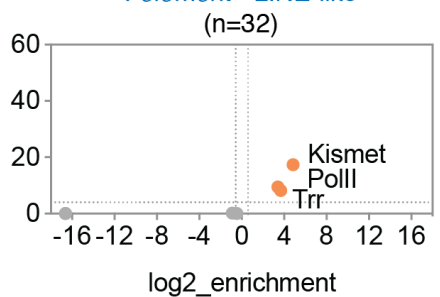

Figure 6. TEs frequently insert in genes and regions of open enhancer-like chromatin 
(A) The distribution of somatic TE insertion sites on the Drosophila chromosomes. Dark grey boxes represent heterochromatic regions. (B) Somatic TE insertion sites were depleted from intergenic and exonic sequences and enriched in introns and 3'UTR regions of the fly genome. (C) Selected genes relevant for the gut physiology with putative somatic insertion sites. Genic regions +/- 500bp were considered. (D) Correlations of somatic insertion sites of the three most represented TE families (rover, copia and I-element) with modENCODE tracks for adult fly tissues. (E) Correlations of somatic insertion sites of the three most represented TE families (rover, copia and I-element) with DamID tracks for adult fly intestinal stem cells (ISC). Colored data points and labels highlight significant positive or negative correlations $(p<0.0001,-1.5>$ enrichment $>1.5)$. de novo insertions from the Pros $>2 X G F P$ clonal gut samples obtained with the short-read sequencing were used for all plots. 